Acta Appl.Math. 28, N1,(1992), 1-42.

\title{
STABILITY ESTIMATES IN INVERSE SCATTERING
}

A. G. Ramm, Mathematics Department,

Kansas State University, Manhattan, KS 66506-2602, USA

Abstract: An algorithm is given for calculating the solution to the 3D inverse scattering problem with noisy discrete fixed energy data. The error estimates for the calculated solution are derived. The methods developed are of general nature and can be used in many applications: in nondestructive evaluation and remote sensing, in geophysical exploration, medical diagnostics and technology.

\section{Introduction}

Let $q \in Q:=\left\{q: q(x)=0\right.$ for $\left.|x| \geq a, \quad x \in R^{3}, \quad q(x)=\bar{q}(x), \quad q \in L^{\infty}\right\}, \quad a>0$ is a constant and the bar stands for complex conjugate. Consider the equation

$$
l_{q} u:=\left[\nabla^{2}+1-q(x)\right] u(x, \alpha)=0 \text { in } R^{3}, \quad \alpha \in S^{2}
$$

where $S^{2}$ is the unit sphere in $R^{3}$ and

$$
u(x, \alpha)=\exp (i \alpha \cdot x)+r^{-1} \exp (i r) A\left(\alpha^{\prime}, \alpha\right)+o\left(r^{-1}\right), \quad r=|x| \rightarrow \infty, \quad \alpha^{\prime}=x / r
$$

The existence and uniqueness of the solution to (1), (2) are well known and are easy to prove (see e.g. [1,2]). The coefficient $A\left(\alpha^{\prime}, \alpha\right)$ is called the scattering amplitude at the fixed energy $\left(k^{2}=1\right)$. Its properties are discussed e.g. in [2], pp. 219-246. The inverse scattering problem (ISP) consists of finding $q \in Q$ from the knowledge of $A\left(\alpha^{\prime}, \alpha\right)$ for all $\alpha^{\prime}, \alpha \in S^{2}$. Uniqueness of the solution to ISP is proved in [3,4], (see also [2]) even in the case when $A\left(\alpha^{\prime}, \alpha\right)$ is known for $\alpha \in \tilde{S}_{1}^{2}, \alpha^{\prime} \in \tilde{S}_{2}^{2}$, where $\tilde{S}_{j}^{2}, j=1,2$, are arbitrary small open sets in $S^{2}$.

The uniqueness result holds with the same proof as in [4] for the problem

$$
\left[\nabla^{2}+1-q_{0}(x)-q(x)\right] u=0 \text { in } R^{3} \text { and }(1.2) \text { holds }
$$

where $q_{0}(x)$ is a known short-range potential, e.g. $\left|q_{0}(x)\right| \leq c(1+|x|)^{-2-\varepsilon}, \varepsilon>0$ and $q(x) \in Q$ (this was noticed by R. Weder, in a preprint, 1991). The aim of this paper is to complete the study of the stability of the solution to ISP with noisy data which the author carried over in the papers [4-18] and in the monograph [1]. The statement of this problem $\left(\mathrm{ISP}_{\delta}\right)$ is as follows.

Let a function $A_{\delta}\left(\alpha^{\prime}, \alpha\right)$ be given which is not assumed to be a scattering amplitude corresponding to a potential and let

$$
\sup _{\alpha^{\prime}, \alpha \in S^{2}}\left|A_{\delta}\left(\alpha^{\prime}, \alpha\right)-A\left(\alpha^{\prime}, \alpha\right)\right|<\delta
$$

The inverse scattering problem with noisy data is: $\left(\operatorname{ISP}_{\delta}\right)$ : Given $A_{\delta}\left(\alpha^{\prime}, \alpha\right)$ for all $\alpha^{\prime}, \alpha \in S^{2}$ find $\hat{q}_{\delta}(x)$ such that

$$
\sup _{\lambda \in R^{3}}\left|\hat{q}_{\delta}-\tilde{q}(\lambda)\right|<\eta(\delta) \rightarrow 0 \text { as } \delta \rightarrow 0
$$

where

$$
\tilde{q}(\lambda):=\int_{R^{3}} \exp (-i \lambda \cdot x) q(x) d x .
$$

In other words, we want to calculate a stable approximation to $\tilde{q}(\lambda)$ and estimate the rate of decay of $\eta(\delta)$ as $\delta \rightarrow 0$. To get such an estimate uniform in $q$ belonging to a compact subset of $Q$ we assume that

$$
q \in \mathcal{B}_{C}:=\left\{q:\|q\|_{L^{\infty}}+\|\nabla q\|_{L^{\infty}} \leq C\right\}
$$

Key words and phrases: Inverse scattering, stability, noisy data AMS Mathematics subject classification 35R30 (1991 Revision) 
$\mathrm{The} \operatorname{ISP}_{\delta}$ is of considerable practical interest: it is a theoretical basis for many problems in nondestructive evaluation and remote sensing, including, to mention a few, problems of geophysical exploration, medical diagnostics, technology, etc. To the author's knowledge, there were no results concerning three-dimensional $\mathrm{ISP}_{\delta}$ except those given in the works $[1,4-18]$

The basic results of this paper are new. They are formulated as Theorems A, B and C in Section II. Theorem A gives a rigorous inversion procedure for ISP with exact data and an error estimate for this procedure. Theorem $\mathrm{B}$ gives a rigorous contruction of the solution to $\mathrm{ISP}_{\delta}$, and an estimate for the difference between this solution and the unknown potential. Theorem B is of practical interest and can be used in designing a numerical code for solving $\mathrm{ISP}_{\delta}$. An alternative method is given in Section VII.3. Theorem C gives an estimate for the difference of the Fourier transforms of two potentials if an estimate for the difference of the corresponding scattering amplitudes is given at a fixed energy.

There are other new results in this paper. They are formulated as lemmas used in the proof of the basic results. Although the ideas and the techniques from works [4-18] are used in this paper there are many improvements, simplifications in the arguments and the paper is essentially self-contained: no prior knowledge of the author's work is assumed. The results may be of interest to mathematicians, engineers, physicists and numerical analysts interested in $\operatorname{ISP}_{\delta}$.

The paper is organized as follows: Section II contains the statement of the basic results and a number lemmas. Section III contains auxiliary results and proofs of Theorem A and lemmas 10 through 17. Section IV contains proofs of lemmas 1 through 9 except lemma 6, which is proved in Section V. In Section VI proofs of Theorems B and C are given. In Section VII some numerical aspects of the $\mathrm{ISP}_{\delta}$ are discussed and a summary of the numerical approach is given. In addition, an alternative numerical method for solving $\mathrm{ISP}_{\delta}$ is discussed and an error estimate for this method is proved.

\section{Formulation of the results}

1. First, assume that the exact data are given:

$$
A\left(\alpha^{\prime}, \alpha\right)=\sum_{\ell=0}^{\infty} A_{\ell}(\alpha) Y_{\ell}\left(\alpha^{\prime}\right), \quad A_{\ell}(\alpha):=\int_{S^{2}} A\left(\alpha^{\prime}, \alpha\right) \bar{Y}_{\ell}\left(\alpha^{\prime}\right) d \alpha^{\prime}
$$

Here and below the summation in $\ell$ denotes $\sum_{\ell=0}^{\infty} \sum_{m=-\ell}^{\ell}, \quad A_{\ell}(\alpha)=A_{\ell m}(\alpha), \quad Y_{\ell}(\alpha)=Y_{\ell m}(\alpha)$, $-\ell \leq m \leq \ell$,

$$
Y_{\ell m}(\alpha)=\frac{1}{\sqrt{4 \pi}}\left[\frac{(2 \ell+1)(\ell-|m|) !}{(\ell+|m|) !}\right]^{1 / 2} P_{\ell,|m|}(\cos \vartheta) \exp (i m \phi)
$$

where $(\vartheta, \phi)$ are the angles in the spherical coordinates determining the unit vector $\alpha \in S^{2}$ :

$$
\alpha_{1}=\sin \vartheta \cos \phi, \quad \alpha_{2}=\sin \vartheta \sin \phi, \quad \alpha_{3}=\cos \vartheta
$$

Define

$$
\begin{gathered}
M:=\left\{\theta: \theta \in C^{3}, \quad \theta \cdot \theta=1\right\}, \\
\theta \cdot \omega=\theta_{1} \cdot \omega_{1}+\theta_{2} \cdot \omega_{2}+\theta_{3} \cdot \omega_{3}, \quad|\theta|=(\theta \cdot \bar{\theta})^{1 / 2}
\end{gathered}
$$

If $0 \leq \vartheta \leq \pi, \quad 0 \leq \phi<2 \pi$, then the vector $\alpha=\left(\alpha_{1}, \alpha_{2}, \alpha_{3}\right)$ runs through $S^{2}$. If $\vartheta, \phi$ run through the complex plane $C$ then the corresponding $\alpha$ runs through $M^{\prime} \subset M$. The subset $M^{\prime}$ contains all the vectors of $M$ except the ones of the form $\left(v_{1}, v_{2}, 1\right)$ and $\left(v_{1}, v_{2},-1\right)$, where $v:=\left(v_{1}, v_{2}\right) \in C^{2}$ are vectors with the property $v \cdot v=0$, and $v \neq 0$. From (2.2) it follows that $Y_{\ell m}(\alpha)$ is defined for all $\alpha \in M^{\prime}$ : it is sufficient to find for $\alpha \in M^{\prime}$ the corresponding complex numbers $\vartheta$ and $\phi$ such that formulas (2.3) hold and calculate the right-hand side of (2.2) for these $\vartheta$ and $\phi$. Obviously $\exp (i m \phi)$ is defined for complex $\phi$, and the function

$$
P_{\ell,|m|}(\cos \vartheta)=(\sin \vartheta)^{|m|} \frac{d^{|m|} P_{\ell}(\cos \vartheta)}{(d \cos \vartheta)^{m}}
$$

is defined for complex $\vartheta$. Let

$$
B_{a}:=\left\{x: x \in R^{3}, \quad|x| \leq a\right\}, \quad Q_{a}:=\left\{q: q=\bar{q}, \quad q=0 \text { for }|x|>a, \quad q \in L^{2}\left(B_{a}\right)\right\} .
$$


Lemma 1: If $q \in Q_{a}$ then

$$
\sup _{\substack{\alpha \in S^{2} \\-\ell \leq m \leq \ell}}\left|A_{\ell m}(\alpha)\right| \leq c a\left(\frac{a e}{2 \ell+1}\right)^{\frac{2 \ell+1}{2}} \frac{1}{2 \ell+1}
$$

Lemma 2: If $\theta \in M^{\prime}$ then

$$
\left|Y_{\ell}(\theta)\right| \leq \frac{1}{\sqrt{4 \pi}} \frac{\exp (\kappa r)}{\left|j_{\ell}(r)\right|}, \quad r>0, \quad \kappa:=|\operatorname{Im} \theta|
$$

where $r>0$ is an arbitrary fixed number and $j_{\ell}(r)$ is the spherical Bessel function, $j_{\ell}(r):=[\pi /(2 r)]^{1 / 2} J_{\ell+\frac{1}{2}}(r), \quad J_{\ell+\frac{1}{2}}(r)$ is the usual Bessel function.

It is well known that

$$
j_{\ell}(r)=\frac{1}{\sqrt{2 r}}\left(\frac{e r}{2 \ell+1}\right)^{\frac{2 \ell+1}{2}} \frac{1}{\sqrt{2 \ell+1}}[1+o(1)] \text { as } l \rightarrow \infty
$$

uniformly in $r \in[0, a]$ for any fixed $a>0$. From (2.7)-(2.9) it follows that the series

$$
A\left(\theta^{\prime}, \alpha\right)=\sum_{\ell=0}^{\infty} A_{\ell}(\alpha) Y_{\ell}\left(\theta^{\prime}\right), \quad \theta^{\prime} \in M, \quad \alpha \in S^{2}
$$

converges absolutely and uniformly on $\tilde{M} \times S^{2}$, where $\tilde{M} \subset M^{\prime}$ is an arbitrary compact subset in $M^{\prime}$. Indeed, choose $r>a$ in (2.8). Then the series (2.10) is majorized by the convergent series

$$
c \exp (\kappa r) \sum_{\ell=0}^{\infty} \sqrt{2 \ell+1}\left(\frac{a}{r}\right)^{\frac{2 \ell+1}{2}}
$$

Note that given the data $A\left(\alpha^{\prime}, \alpha\right) \forall \alpha^{\prime}, \alpha \in S^{2}$ one finds the Fourier coefficients $A_{\ell}(\alpha)$ by formula (2.1) and defines $A\left(\theta^{\prime}, \alpha\right) \forall \theta^{\prime} \in M, \alpha \in S^{2}$ by formula (2.10).

2. We now pass to the description of the inversion formula for exact data. Define

$$
\rho(\nu):=\exp (-i \theta \cdot x) \int_{S^{2}} u(x, \alpha) \nu(\alpha) d \alpha-1, \quad \theta \in M^{\prime}, \quad \nu \in L^{2}\left(S^{2}\right)
$$

Consider the variational problem

$$
\|\rho(\nu)\|:=\inf :=d(\theta)
$$

where $\rho(\nu)$ is a function of $x \in R^{3}$ and $\theta \in M^{\prime}$,

$$
\|\rho\|:=\|\rho\|_{L^{2}\left(B_{b} \backslash B_{a_{1}}\right)}, \quad a<a_{1}<b
$$

and $a_{1}, b$ are arbitrary numbers subject to the restriction (2.14). Note that in the annulus $a_{1}<|x|<b$ the scattering solution $u(x, \alpha)$ is defined explicitly through the data. Namely the following simple lemma holds: Lemma 3: One has

$$
u(x, \alpha)=\exp (i \alpha \cdot x)+\sum_{\ell=0}^{\infty} A_{\ell}(\alpha) Y_{\ell}\left(\alpha^{\prime}\right) h_{\ell}(r), \quad r>a, \quad r=|x|, \quad \alpha^{\prime}=x / r
$$

where $h_{\ell}(r)$ are the spherical Hankel functions normalized so that $h_{\ell}(r) \sim r^{-1} \exp (i r)$ as $r \rightarrow \infty$.

Lemma 4: If $q \in Q_{a}$ then

$$
d(\theta) \leq c|\theta|^{-1}, \quad|\theta| \gg 1, \quad \theta \in M^{\prime}
$$


Here and below $c$ stand for various positive constants which do not depend on $\theta$ and $\delta$. These constants depend on the norm $\|q\|_{a}:=\|q\|_{L^{2}\left(B_{a}\right)}$ for $q \in Q$, and for $q \in \mathcal{B}_{C}$ they depend on $C$ where $C$ is defined in (1.5). The notation $|\theta| \gg 1$ means that $\theta$ is sufficiently large.

Denote by $\nu(\alpha, \theta)$ an arbitrary function in $L^{2}\left(S^{2}\right)$ which satisfies the inequality

$$
\|\rho(\nu(\alpha, \theta))\| \leq(c+1)|\theta|^{-1}, \quad|\theta| \rightarrow \infty
$$

It follows from Lemma 4 that such a function can be calculated by solving problem (2.13). Define for this $\nu(\alpha, \theta)$ the quantity

$$
\hat{q}:=-4 \pi \int_{S^{2}} A\left(\theta^{\prime}, \alpha\right) \nu(\alpha, \theta) d \alpha, \quad \theta^{\prime}, \theta \in M^{\prime}
$$

Fix an arbitrary large number $\lambda_{0}>0$, take any $\lambda \in R^{3},|\lambda| \leq \lambda_{0}$, and pick any $\theta^{\prime}, \theta$ such that

$$
\theta^{\prime}-\theta=\lambda, \quad \theta^{\prime}, \theta \in M, \quad|\theta| \rightarrow \infty
$$

Lemma 5: For any $\lambda \in R^{n}, n \geq 3$ there exist $\theta, \theta^{\prime} \in M$ satisfying (2.19).

Remark: For $n=2$ this is not true as one can easily check. If $\lambda_{3} \neq \pm 1$ then one can find $\theta, \theta^{\prime} \in M^{\prime}$ satisfying (2.19). In what follows we may assume without loss of generality that $\lambda_{3} \neq \pm 1$ and use $M^{\prime}$ everywhere. Indeed, we can find the Fourier transform of the potential, $\tilde{q}(\lambda)$ for any $\lambda$ with $\lambda_{3} \neq \pm 1$ and since $\tilde{q}$ is continuous, in fact analytic, with respect to $\lambda$, the value of $\tilde{q}$ at $\lambda=(0,0, \pm 1)$ is determined uniquely by the continuity of $\tilde{q}$.

Let

$$
\tilde{q}(\lambda):=\int_{B_{a}} q(x) \exp (-i \lambda \cdot x) d x
$$

3. We are ready to formulate the first theorem.

Theorem A: If $q \in Q, \hat{q}$ is defined by (2.18) and (2.17), (2.19) hold, then

$$
\sup _{|\lambda| \leq \lambda_{0}}|\tilde{q}(\lambda)-\hat{q}| \leq c|\theta|^{-1}, \quad|\theta| \rightarrow \infty
$$

The constant $c$ in (2.21) can be chosen uniformly in $q \in \mathcal{B}_{C}$ where $\mathcal{B}_{C}$ is defined in (1.5).

Corollary: The following inversion formula holds

$$
\tilde{q}(\lambda)=-4 \pi \lim _{|\theta| \rightarrow \infty} \int_{S^{2}} A\left(\theta^{\prime}, \alpha\right) \nu(\alpha, \theta) d \alpha, \quad \theta^{\prime}-\theta=\lambda, \quad \theta^{\prime}, \theta \in M^{\prime}
$$

Estimate (2.21) is the error estimate for the inversion formula (2.18) for the exact data $A\left(\alpha^{\prime}, \alpha\right)$. Note that as the data we actually use the coefficients $A_{\ell}(\alpha), \ell \geq 0$. In the proof of Theorem A the following major lemma is used:

Lemma 6: If $\rho$ is defined by (2.12) and $|\theta| \gg 1, \theta \in M$, then

$$
\|\rho\|_{a} \leq c\left(\|\rho\|+|\theta|^{-1}\right), \quad\|\cdot\|_{a}:=\|\cdot\|_{L^{2}\left(B_{a}\right)}
$$

4. We will turn now to the description of the inversion formulas for noisy data $A_{\delta}\left(\alpha^{\prime}, \alpha\right)$ given for all $\alpha^{\prime}, \alpha \in S^{2}$. It will become clear later that we actually can use only a discrete subset of noisy data $A_{\delta}\left(\alpha_{j}^{\prime}, \alpha_{p}\right)$ for some $\alpha_{j}^{\prime}, \alpha_{p} \in S^{2}$. Let $[x]$ denote the integer nearest to the real number $x$. Define

$$
N(\delta):=\left[\frac{|\ln \delta|}{\ln |\ln \delta|}\right]
$$




$$
\begin{gathered}
\hat{A}_{\delta}\left(\theta^{\prime}, \alpha\right):=\sum_{\ell=0}^{N(\delta)} A_{\delta \ell}(\alpha) Y_{\ell}\left(\theta^{\prime}\right), \quad A_{\delta \ell}(\alpha):=\int_{S^{2}} A_{\delta}\left(\alpha^{\prime}, \alpha\right) \overline{Y_{\ell}\left(\alpha^{\prime}\right)} d \alpha^{\prime} \\
u_{\delta}(x, \alpha):=\exp (i \alpha \cdot x)+\sum_{\ell=0}^{N(\delta)} A_{\delta \ell}(\alpha) Y_{\ell}\left(\alpha^{\prime}\right) h_{\ell}(r) \\
\rho_{\delta}(\nu):=\exp (-i \theta \cdot x) \int_{S^{2}} u_{\delta}(x, \alpha) \nu(\alpha) d \alpha-1, \quad \theta \in M^{\prime} \\
\mu(\delta)=\exp [-\gamma N(\delta)], \quad \gamma:=\ln \frac{a_{1}}{a}>0 \\
a(\nu):=\|\nu\|_{L^{2}\left(S^{2}\right)}
\end{gathered}
$$

Lemma 7: One has, with $\kappa:=|\operatorname{Im} \theta|$,

$$
\begin{gathered}
\left\|\rho_{\delta}(\nu)\right\| \leq\|\rho(\nu)\|+c a(\nu) \exp (\kappa b) \mu(\delta), \\
\|\rho(\nu)\| \leq\left\|\rho_{\delta}(\nu)\right\|+c a(\nu) \exp (\kappa b) \mu(\delta) .
\end{gathered}
$$

Consider the variational problem

$$
|\theta|=\sup :=\Theta(\delta), \quad\left\|\rho_{\delta}(\nu)\right\|+a(\nu) \exp (\kappa b) \mu(\delta) \leq c|\theta|^{-1}, \quad \theta \in M^{\prime}
$$

where $c>0$ is a sufficiently large fixed constant, the supremum is taken over $\nu \in L^{2}\left(S^{2}\right)$ and $\theta \in M^{\prime}, \delta>0$ being fixed.

\section{Lemma 8:}

$$
\Theta(\delta) \rightarrow \infty \text { as } \delta \rightarrow 0
$$

See also formula (4.39) for a stronger result.

Let $\nu_{\delta}(\alpha) \in L^{2}\left(S^{2}\right)$ be any function such that

$$
\left\|\rho_{\delta}(\nu)\right\|+a\left(\nu_{\delta}\right) \exp (\kappa b) \mu_{1}(\delta) \leq c|\theta(\delta)|^{-1}, \quad|\theta(\delta)|>\frac{\Theta(\delta)}{2}
$$

where $\kappa=\kappa(\delta):=|\operatorname{Im} \theta(\delta)|$. One can calculate such $\nu_{\delta}(\alpha)$ and $\theta(\delta)$ by solving problem (2.32). Find $\theta^{\prime}(\delta) \in M^{\prime}$ such that (2.19) holds with an arbitrary fixed $\lambda \in R^{3},|\lambda| \leq \lambda_{0}$. Define

$$
\hat{q}_{\delta}:=-4 \pi \int_{S^{2}} \hat{A}_{\delta}\left(\theta^{\prime}(\delta), \alpha\right) \nu_{\delta}(\alpha) d \alpha
$$

Our main result is an error estimate for the inversion formula (2.35) for noisy data. We assume that (1.3) holds and $q \in Q$ where $Q$ is defined in Section I.

Theorem B: If $q \in Q$ and (1.3) holds, then

$$
\sup _{|\lambda| \leq \lambda_{0}}\left|\tilde{q}(\lambda)-\hat{q}_{\delta}\right| \leq c|\theta(\delta)|^{-1}
$$

where $\hat{q}_{\delta}$ and $\tilde{q}(\lambda)$ are defined in (2.35) and (2.20),

$$
|\theta(\delta)| \geq \frac{|\ln \delta|}{(\ln |\ln \delta|)^{2}},
$$


the constant $c$ in (2.36) does not depend on $\delta$ and can be chosen uniformly for $q \in \mathcal{B}_{C}$.

5 . If one applies a quadrature formula to (2.35) one obtains

$$
\hat{q}_{\delta} \approx-4 \pi \sum_{j=1}^{n} \hat{A}_{\delta}\left(\theta^{\prime}(\delta), \alpha_{j}\right) \nu_{\delta}\left(\alpha_{j}\right) c_{j}
$$

where $\alpha_{j}$ are the nodes and $c_{j}$ are the coefficients of a quadrature formula. Formula (2.38) uses discrete noisy data. Using the definition (2.25) and applying a quadrature formula again one has

$$
\hat{A}_{\delta \ell}\left(\alpha_{j}\right) \approx \sum_{p=1}^{n_{1}} A_{\delta}\left(\alpha_{p}^{\prime}, \alpha_{j}\right) \overline{Y_{\ell}\left(\alpha_{p}^{\prime}\right)} c_{p}^{\prime}
$$

Combining (2.35), (2.25), (2.38) and (2.39) one gets

$$
\hat{q} \approx-4 \pi \sum_{j=1}^{n} \nu_{\delta}\left(\alpha_{j}\right) c_{j} \sum_{\ell=0}^{N(\delta)} Y_{\ell}\left(\theta^{\prime}(\delta)\right) \sum_{p=1}^{n_{1}} A_{\delta}\left(\alpha_{p}^{\prime}, \alpha_{j}\right) \overline{Y_{\ell}\left(\alpha_{p}^{\prime}\right)} c_{p}^{\prime}
$$

This formula uses the discrete noisy data $A_{\delta}\left(\alpha_{p}^{\prime}, \alpha_{j}\right), 1 \leq p \leq n_{1}, 1 \leq j \leq n$. In principle one can estimate the error of the quadrature formulas $(2.37)-\left(2.39^{\prime}\right)$ in terms of some bounds on the derivatives of $\hat{A}_{\delta}\left(\theta^{\prime}(\delta), \alpha\right)$ and $\nu_{\delta}(\alpha)$, but we do not go into detail.

6. Let us consider the following question. Suppose $q_{j} \in Q, j=1,2, A_{j}\left(\alpha^{\prime}, \alpha\right)$ is the scattering amplitude corresponding to the potential $q_{j}$. The question is: how does one estimate $p:=q_{1}-q_{2}$ in terms of $A:=A_{1}-A_{2}$. The following lemma is useful.

Lemma 9: One has

$$
-4 \pi A\left(\alpha^{\prime}, \alpha\right)=\int_{B_{a}} p(x) u_{1}(x, \alpha) u_{2}\left(x,-\alpha^{\prime}\right) d x
$$

where $u_{j}(x, \alpha)$ is the scattering solution corresponding to the potential $q_{j}(x), j=1,2$.

The answer to the above question is given in the following theorem.

Theorem C: If

$$
\sup _{\alpha^{\prime}, \alpha \in S^{2}}\left|A_{1}\left(\alpha^{\prime}, \alpha\right)-A_{2}\left(\alpha^{\prime}, \alpha\right)\right|<\delta
$$

then

$$
\sup _{|\lambda| \leq \lambda_{0}}|\tilde{p}(\lambda)| \leq c \frac{\ln |\ln \delta|}{|\ln \delta|}
$$

where $c=$ const $>0$ can be chosen uniformly for $q \in \mathcal{B}_{C}$.

\section{Auxiliary Results and Proofs of Theorem A and Lemmas 10-17}

1. In this section we give some auxiliary results which are of independent interest and also help to prove the results formulated in Theorems A-C.

Let us start with an estimate of the quantity $\tilde{q}(\lambda)$. The starting point is the well-known formula

$$
-4 \pi A\left(\theta^{\prime}, \alpha\right)=\int_{B_{a}} \exp \left(-i \theta^{\prime} \cdot x\right) u(x, \alpha) q(x) d x
$$

Multiply (3.1) by $\nu(\alpha) \in L^{2}\left(S^{2}\right)$ and integrate over $S^{2}$. Write the result as

$$
-4 \pi \int_{S^{2}} A\left(\theta^{\prime}, \alpha\right) \nu(\alpha) d \alpha=\int_{B_{a}} \exp \left[-i\left(\theta^{\prime}-\theta\right) \cdot x\right][1+\rho(\nu)] q(x) d x
$$

Here $\rho(\nu)$ is defined in (2.12), $\theta^{\prime}$ and $\theta$ satisfy (2.19), and (3.2) can be written as

$$
\left|-4 \pi \int_{S^{2}} A\left(\theta^{\prime}, \alpha\right) \nu(\alpha) d \alpha-\tilde{q}(\lambda)\right|=\left|\int_{B_{a}} \exp (-i \lambda \cdot x) q(x) \rho(\nu) d x\right| \leq\|q\|_{a}\|\rho(\nu)\|_{a}
$$


where $\tilde{q}(\lambda)$ is defined $(2.20)$ and $\|\cdot\|_{a}=\|\cdot\|_{L^{2}\left(B_{a}\right)}$.

Let us formulate the result:

Lemma 10: If $q \in Q_{a}, \nu \in L^{2}\left(S^{2}\right)$ and (2.19) holds then (3.3) holds.

Proof of Theorem A: If one uses (3.3), chooses $\nu=\nu(\alpha, \theta)$ such that (2.17) holds, and uses (2.23), one obtains formula (2.21), and (2.22) follows from (2.21). Theorem A is proved.

2. In the proof of Theorem B the following auxiliary results are important.

Lemma 11: One has

$$
\begin{gathered}
\left|\hat{q}_{\delta}-\tilde{q}(\lambda)\right| \leq 4 \pi\left\|\hat{A}_{\delta}\left(\theta^{\prime}, \alpha\right)-A\left(\theta^{\prime}, \alpha\right)\right\|_{L^{2}\left(S^{2}\right)} a\left(\nu_{\delta}\right)+\left\|\rho\left(\nu_{\delta}\right)\right\| a\|q\|_{a}, \\
\left\|\hat{A}_{\delta}\left(\theta^{\prime}, \alpha\right)-A\left(\theta^{\prime}, \alpha\right)\right\|_{L^{2}\left(S^{2}\right)} \leq c \exp \left(\kappa a_{1}\right) \mu(\delta)<c \exp (\kappa b) \mu(\delta),
\end{gathered}
$$

where $\hat{A}_{\delta}\left(\theta^{\prime}, \alpha\right)$ is given by (2.25), $A\left(\theta^{\prime}, \alpha\right)$ is given by (2.10), $\theta^{\prime}(\delta)$ and $\theta(\delta)$ satisfy (2.19),

$$
\begin{gathered}
\kappa:=|\operatorname{Im} \theta(\delta)|, \quad \mu(\delta):=\ln [-\gamma N(\delta)], \quad \gamma:=\ln \frac{a_{1}}{a}>0, \\
a\left(\nu_{\delta}\right):=\left\|\nu_{\delta}\right\|_{L^{2}\left(S^{2}\right)}
\end{gathered}
$$

$\tilde{q}(\lambda)$ and $\hat{q}_{\delta}$ are defined in (2.20) and (2.35), and $N(\delta)$ is defined by (2.24).

We collect proofs at the end of this section.

3. In the proof of Theorems $\mathrm{B}$ and $\mathrm{C}$ the following results are used. They are of independent interest.

Lemma 12: If $q \in Q_{a}, \theta \in M,|\theta| \gg 1$ then there exists $\psi$ which satisfies the equation

$$
\ell_{q} \psi=0 \text { in } R^{3}, \quad \psi=\exp (i \theta \cdot x)[1+R(x, \theta)]
$$

and

$$
\|R\|_{L^{2}(D)} \leq c|\theta|^{-1}, \quad|\theta| \rightarrow \infty
$$

where $D \subset R^{3}$ is an arbitrary bounded region, the constant $c$ in (3.7) depends on diam $D$ and on $\|q\|_{a}:=$ $\|q\|_{L^{2}\left(B_{a}\right)}$.

The following estimate holds [6] :

$$
\|R\|_{L^{\infty}(D)}<c \frac{(\ln |\theta|)^{\frac{1}{2}}}{|\theta|^{\frac{1}{2}}}
$$

Let $\ell:=\ell_{q}, N_{D}(\ell):=\left\{w: \ell w=0\right.$ in $\left.D, w \in H^{2}(D)\right\}$, where $H^{m}(D)$ is the usual Sobolev space, $D \subset R^{3}$ is an arbitrary bounded region, with a smooth boundary, homeomorphic to a ball.

Lemma 13: Let $w \in N_{D}(\ell)$ and $\varepsilon>0$ be an arbitrary small number. Then there exists $\nu_{\varepsilon}(\alpha)$ such that

$$
\left\|w-\int_{S^{2}} u(x, \alpha) \nu_{\varepsilon}(\alpha) d \alpha\right\|_{L^{2}(D)}<\varepsilon
$$

Here $u(x, \alpha)$ is the scattering solution defined in (1.1), (1.2).

Lemma 14: Suppose $w=\psi$ and (3.8) holds with $w=\psi, \operatorname{Im} \theta \neq 0$. Then

$$
\begin{gathered}
\lim _{\varepsilon \rightarrow 0}\left\|\nu_{\varepsilon}(\alpha)\right\|=\infty, \\
\left\|\nu_{\varepsilon}\right\| \geq c \exp (\kappa d / 2), \quad \kappa:=|\operatorname{Im} \theta|, \quad d=\operatorname{diam} D
\end{gathered}
$$

Suppose that

$$
\left\|\psi(x, \theta)-\int_{S^{2}} u(x, \alpha) \nu_{\varepsilon}(\alpha) d \alpha\right\| \leq \varepsilon
$$


where the norm in (3.11) is defined in (2.14). This norm is not to be confused with the norm in $L^{2}\left(S^{2}\right)$. It is clear from the context what norm is meant.

Consider the problem of finding

$$
\inf \left\|\nu_{\varepsilon}(\alpha)\right\|:=j(\varepsilon, \theta)
$$

where the infimum is taken over the functions $\nu_{\varepsilon}(\alpha) \in L^{2}\left(S^{2}\right)$ which satisfy (3.11).

If $\theta, \theta \in M$, is arbitrary large fixed, $\varepsilon>0$, and

$$
n(\varepsilon)=\exp \left\{\left(\ln \ln \varepsilon^{-1}\right)[1+o(1)]\right\}, \quad \varepsilon \rightarrow 0
$$

then the following estimate holds.

Lemma 15: One has

$$
j(\varepsilon, \theta) \leq c \exp (\kappa r)\left(\frac{2 n(\varepsilon)+1}{e r}\right)^{n(\varepsilon)} n^{2}(\varepsilon), \quad r>b, \quad \kappa=|\operatorname{Im} \theta|
$$

where $r>b$ is arbitrary and $c$ does not depend on $\varepsilon$ and $\theta$.

Corollary: Minimizing in $r>b$ one obtains

$$
j(\varepsilon, \theta) \leq c(2 \kappa)^{n(\varepsilon)} n^{2}(\varepsilon), \quad \kappa=|\operatorname{Im} \theta|
$$

If $N(\kappa)$ is the asymptotic solution to the equation

$$
\left(\frac{e b \kappa}{N}\right)^{N}=c_{2} \frac{\exp (-\kappa b)}{\kappa}, \quad \kappa \rightarrow+\infty
$$

then, with $\varepsilon(\kappa):=\kappa^{-1} \exp (-\kappa b)$, the following estimate holds.

Lemma 16: One has

$$
j(\varepsilon(\kappa), \theta) \leq c N^{3}(\kappa)(2 \kappa)^{2 N(\kappa)+1}, \quad \kappa:=|\operatorname{Im} \theta| \rightarrow \infty,
$$

and $N(\kappa)$ is of the order $\kappa$ as $\kappa \rightarrow \infty$ :

$$
e b \kappa<N(\kappa)<e^{2} b \kappa, \quad \kappa \rightarrow \infty
$$

Thus

$$
j(\varepsilon(\kappa), \theta) \leq c \kappa^{4}(2 \kappa)^{2 e^{2} b \kappa} \leq c \kappa^{4}(2 \kappa)^{20 b \kappa} .
$$

4. Let $q \in \mathcal{B}_{C}$, where $\mathcal{B}_{C}$ is defined in (1.5). The scattering solution satisfies the equation

$$
\left(I+T_{q}\right) u=\exp (i \alpha \cdot x), \quad T_{q} u:=\int_{B_{a}} \frac{\exp (i|x-y|)}{4 \pi|x-y|} q(y) u(y) d y
$$

Consider the operator $I+T_{q}$ as an operator in $L^{2}\left(B_{a}\right)$. Note that $T_{q}: L^{2}\left(B_{a}\right) \rightarrow L^{2}\left(B_{a}\right)$ is compact.

Lemma 17: The operator $I+T_{q}$ is an isomorphism of $L^{2}\left(B_{a}\right)$ onto $L^{2}\left(B_{a}\right)$ and

$$
\sup _{q \in \mathcal{B}_{C}}\left\|\left(I+T_{q}\right)^{-1}\right\| \leq c
$$

where the constant $c$ depends on $C$ and does not depend on $q \in \mathcal{B}_{C}$.

5. The following estimate holds (cf. (2.9)):

$$
\left|j_{\ell}(r)\right| \leq c r^{-1 / 2}\left(\frac{e r}{2 \ell+1}\right)^{\frac{2 \ell+1}{2}} \frac{1}{(2 \ell+1)^{1 / 2}}, \quad 0 \leq r \leq r_{0}, \quad \ell \geq 0
$$


where $j_{\ell}(r)$ is the spherical Bessel function.

6 . Let us prove the lemmas of this section.

Proof of Lemma 11. One has, using (2.35),

$$
\hat{q}_{\delta}=-4 \pi \int_{S^{2}}\left[\hat{A}_{\delta}\left(\theta^{\prime}(\delta), \alpha\right)-A\left(\theta^{\prime}(\delta), \alpha\right)\right] \nu_{\delta}(\alpha) d \alpha-4 \pi \int_{S^{2}} A\left(\theta^{\prime}(\delta), \alpha\right) \nu_{\delta}(\alpha) d \alpha
$$

By (3.3) one obtains from (3.20) the estimate

$$
\begin{aligned}
& \left|\hat{q}_{\delta}-\tilde{q}(\lambda)\right| \leq 4 \pi \int_{S^{2}}\left|\hat{A}_{\delta}\left(\theta^{\prime}(\delta), \alpha\right)-A\left(\theta^{\prime}(\delta), \alpha\right)\right|\left|\nu_{\delta}(\alpha)\right| d \alpha+ \\
& +\|q\|_{a}\left\|\rho\left(\nu_{\delta}\right)\right\|_{a} \leq 4 \pi\left\|\hat{A}_{\delta}\left(\theta^{\prime}(\delta), \alpha\right)-A\left(\theta^{\prime}(\delta), \alpha\right)\right\|\left\|\nu_{\delta}(\alpha)\right\|+\|q\|_{a}\left\|\rho\left(\nu_{\delta}\right)\right\|_{a}
\end{aligned}
$$

This is the estimate (3.4). Let us prove (3.5)

One has

$$
\begin{gathered}
\left\|\hat{A}_{\delta}\left(\theta^{\prime}(\delta), \alpha\right)-A\left(\theta^{\prime}(\delta), \alpha\right)\right\| \leq\left\|\sum_{\ell=0}^{N(\delta)}\left|A_{\delta \ell}(\alpha)-A_{\ell}(\alpha)\right|\left|Y_{\ell}\left(\theta^{\prime}\right)\right|\right\|+ \\
+\left\|\sum_{\ell=N(\delta)+1}^{\infty}\left|A_{\ell}(\alpha)\right|\left|Y_{\ell}(\theta)\right|\right\|:=I_{1}+I_{2}
\end{gathered}
$$

Using estimate (2.8) with $r=a_{1}>a$, and (2.9), and taking into account that $\left|\operatorname{Im} \theta^{\prime}\right|=|\operatorname{Im} \theta|=\kappa$ if $\theta^{\prime}-\theta=\lambda, \lambda \in R^{3}$, one obtains

$$
\begin{aligned}
& I_{1} \leq c \delta \exp \left(\kappa a_{1}\right) N^{2}(\delta)\left[\left(\frac{e a_{1}}{2 N(\delta)+1}\right)^{\frac{2 N(\delta)+1}{2}} \frac{1}{\sqrt{N(\delta)}}\right]^{-1}= \\
& =c \delta \exp \left(\kappa a_{1}\right) N^{5 / 2}(\delta)\left(\frac{2 N(\delta)+1}{e a_{1}}\right)^{\frac{2 N(\delta)+1}{2}} \leq c \delta \exp \left(\kappa a_{1}\right) N^{3}(\delta)\left(\frac{N(\delta)}{e a_{1} / 2}\right)^{N(\delta)}
\end{aligned}
$$

Here we took into account that there are

$$
\sum_{\ell=0}^{N(\delta)}(2 \ell+1)=[N(\delta)+1]^{2}
$$

spherical harmonics with $0 \leq \ell \leq N(\delta)$.

Furthermore, using (2.7), (2.8) and (2.9) one gets

$$
I_{2} \leq \sum_{\ell=N(\delta)+1}^{\infty}\left(\frac{e a}{2 \ell+1}\right)^{\frac{2 \ell+1}{2}} \frac{1}{2 \ell+1} \frac{\exp \left(\kappa a_{1}\right)}{\left(\frac{e a_{1}}{2 \ell+1}\right)^{\frac{2 \ell+1}{2}} \frac{1}{\sqrt{2 \ell+1}}} \leq c N^{1 / 2}(\delta)\left(\frac{a}{a_{1}}\right)^{N(\delta)} \exp \left(\kappa a_{1}\right)
$$

Combining (3.23) and (3.24) one has, with $N=N(\delta), a / a_{1}:=s, 0<s<1, e a_{1} / 2:=t$,

$$
I_{1}+I_{2} \leq c \exp \left(\kappa a_{1}\right)\left\{\delta N^{3}\left(\frac{N}{t}\right)^{N}+N^{1 / 2} s^{N}\right\}
$$

Consider, for a fixed small $\delta>0$, the minimization problem

$$
\delta N^{3}\left(\frac{N}{t}\right)^{N}+N^{1 / 2} s^{N}=\inf
$$


where the infimum is taken over $N, N \gg 1$, that is for sufficiently large $N$. Write (3.26) as

$$
\delta \exp (N \ln N-N \ln t+3 \ln N)+\exp \left(N \ln s+\frac{1}{2} \ln N\right)=\inf
$$

To find the infimum in $N$, let us differentiate (3.27) with respect to $N$ and equate the resulting expression to zero. This yields

$$
\delta \exp (N \ln N-N \ln t+3 \ln N) \ln N\left[1+O\left(\frac{1}{\ln N}\right)\right]+\exp \left(N \ln s+\frac{1}{2} \ln N\right)\left[\ln s+\frac{1}{2 N}\right]=0
$$

Thus

$$
\begin{gathered}
\delta^{-1}=\exp \left(N \ln N-N \ln t+3 \ln N-N \ln s-\frac{1}{2} \ln N\right) \frac{\ln N\left[1+O\left(\frac{1}{\ln N}\right)\right]}{-\frac{1}{2 N}+\ln s^{-1}} \\
\ln \frac{1}{\delta}=N \ln N\left[1+O\left(\frac{1}{\ln N}\right)\right], \quad N \rightarrow \infty \\
\ln \ln \frac{1}{\delta}=\ln N+\ln \ln N+O\left(\frac{1}{\ln N}\right)=(\ln N)\left[1+O\left(\frac{\ln \ln N}{\ln N}\right)\right]
\end{gathered}
$$

Divide (3.30) by (3.31) to obtain

$$
N=N(\delta):=\frac{\ln \frac{1}{\delta}}{\ln \ln \frac{1}{\delta}}[1+o(1)], \quad \delta \rightarrow 0
$$

Denote by $\tilde{\mu}(\delta)$ the infimum in (3.27) which is the value of the left-hand side of (3.27) at $N=N(\delta)$. Then, using (3.27) and (3.28), one gets, with $\gamma:=\ln \left(s^{-1}\right)$,

$$
\tilde{\mu}(\delta)=\exp \{-\gamma N(\delta)[1+o(1)]\}\left\{1+\frac{\gamma-\frac{1}{2 N(\delta)}}{[\ln N(\delta)]\left[1+O\left(\frac{1}{\ln N(\delta)}\right)\right]}\right\} \leq c \mu(\delta),
$$

where $\mu(\delta):=\exp [-\gamma N(\delta)]$.

From (3.32), (3.25) and (3.33) one obtains

$$
I_{1}+I_{2} \leq c \exp \left(\kappa a_{1}\right) \mu(\delta) \leq c \exp (\kappa b) \mu_{1}(\delta)
$$

where $\gamma_{1}:=\ln \left[a_{1} /(a \sqrt{2})\right]\langle\gamma, b\rangle a_{1}$. Therefore estimate (3.5) is proved and the proof of Lemma 11 is complete.

Proof of Lemma 12. Substitute $\psi$ of the form (3.6) into the equation (3.6) to get the equation for $R$ :

$$
L R:=\left(\nabla^{2}+2 i \theta \cdot \nabla\right) R=q(x) R+q(x) \text { in } R^{3}
$$

Define the operator

$$
L^{-1} f:=-\frac{1}{(2 \pi)^{3}} \int_{R^{3}} \frac{\exp (i \lambda \cdot x) \tilde{f}(\lambda)}{\lambda^{2}+2 \lambda \cdot \theta} d \lambda:=w
$$

where

$$
\tilde{f}(\lambda):=\int_{R^{3}} \exp (i \lambda \cdot x) f(x) d x
$$

Then

$$
L w=f \text { in } R^{3}
$$


In Lemma 12 the existence of $\psi$ satisfying (3.6) and (3.7) is claimed. Uniqueness of such a $\psi$ is not discussed. Therefore, Lemma 12 will be proved if one demonstrates the existence of a solution to the equation

$$
R=L^{-1} q R+L^{-1} q \text { in } R^{3}
$$

such that $R$ satisfies (3.7).

Suppose that the following estimate is established

$$
\left\|L^{-1} f\right\|_{L^{2}\left(D_{1}\right)} \leq c|\theta|^{-1}\|f\|_{L^{2}(D)}, \quad \theta \in M, \quad|\theta| \gg 1
$$

for any $f \in L^{2}\left(R^{3}\right)$ vanishing outside of a bounded domain $D, D \subset B_{a} \subset D_{1}, D_{1}$ is an arbitrary bounded domain in $R^{3}$, and $c$ depends on $D$ and $D_{1}$ but not on $\theta$ (we will prove estimate (3.40) later: see estimate (3.47) below). Then equations(3.39) and (3.40) imply, with $\|\cdot\|_{a}=\|\cdot\|_{L^{2}\left(B_{a}\right)}$,

$$
\|R\|_{a} \leq c|\theta|^{-1}\|q R\|_{a}+c|\theta|^{-1}\|q\|_{a} \leq c|\theta|^{-1}\|q\|_{L^{\infty}\left(B_{a}\right)}\|R\|_{a}+c|\theta|^{-1}\|q\|_{a}
$$

Therefore, for $|\theta| \gg 1$ such that $c|\theta|^{-1}\|q\|_{L^{\infty}\left(B_{a}\right)}<1$, one has

$$
\|R\|_{a} \leq c_{1}|\theta|^{-1}\|q\|_{a}
$$

This implies (3.7) under the additional assumption $q \in L^{\infty}\left(B_{a}\right)$.

To complete the proof of Lemma 12 under this additional assumption it is sufficient to prove (3.40). This estimate is an immediate consequence of the following result, which can be found in [0, vol. II, pp. 17, 31]: Let $P(\partial)$ be a partial differential expression with constant coefficients, $\partial:=-i \operatorname{grad}, D \subset R^{n}, n \geq 2, D$ is a bounded domain, $G_{0}(x)$ is a regular fundamental solution: $P(\partial) G_{0}=\delta(x-y), \eta(x) \in C_{0}^{\infty}\left(R^{n}\right), \eta=1$ in a neighborhood of the set $\{x-y\}, x, y \in D_{1}$, and $G_{1}(x-y)=\eta(x-y) G_{0}(x-y)$. Then

$$
\int_{D} G_{1}(x-y) f(y) d y=\int_{D} G_{0}(x-y) f(y) d y, \quad D \subset D_{1}, \quad x \in D_{1}
$$

and

$$
\sup _{\lambda \in R^{n}} \mathcal{P}(\lambda)\left|\tilde{G}_{1}(\lambda)\right|<\infty
$$

where

$$
\mathcal{P}(\lambda):=\left(\sum_{|j| \geq 0}\left|P^{(j)}(\lambda)\right|^{2}\right)^{1 / 2}
$$

and $j$ is a multiindex. If $P(\partial)=L:=\nabla^{2}+2 i \theta \cdot \nabla$, then $L(\lambda)=\lambda^{2}+2 \lambda \cdot \theta$ and

$$
\begin{aligned}
& \mathcal{L}(\lambda)=\left(\sum_{|j| \geq 0}\left|\left(\lambda^{2}+2 \lambda \cdot \theta\right)^{(j)}\right|^{2}\right)^{1 / 2}= \\
= & \left\{\left|\lambda^{2}+2 \lambda \cdot \theta\right|^{2}+4|\lambda+\theta|^{2}+36\right\}^{1 / 2} \geq 2|\operatorname{Im} \theta|
\end{aligned}
$$

Therefore

$$
\begin{aligned}
& \left\|L^{-1} f\right\|_{L^{2}\left(D_{1}\right)}^{2}=\int_{D_{1}}\left|\int_{D} G_{1}(x-y) f(y) d y\right|^{2} d x \leq \frac{1}{(2 \pi)^{3}} \int_{R^{3}} d \lambda\left|\tilde{G}_{1}(\lambda)\right|^{2}|\tilde{f}(\lambda)|^{2} \leq \\
& \leq c \max _{\lambda \in R^{3}}\left[\mathcal{L}^{2}(\lambda)\left|\tilde{G}_{1}(\lambda)\right|^{2}\right]\|f\|_{L^{2}(D)}^{2} \cdot \frac{1}{\min _{\lambda \in R^{3}} \mathcal{L}^{2}(\lambda)} \leq c|\theta|^{-2}\|f\|_{L^{2}(D)}^{2}
\end{aligned}
$$

Estimate (3.47) is identical with (3.40). Therefore Lemma 12 is proved under the additional assumption $q \in L^{\infty}\left(B_{a}\right)$. Without this assumption estimate (3.7) is proved in [6] and in [1]. The argument in [6] is more complicated. It uses estimate (3.7'):

$$
\left\|L^{-1} f\right\|_{L^{\infty}\left(D_{1}\right)} \leq c|\theta|^{-1 / 2}(\ln |\theta|)^{1 / 2}\|f\|_{L^{2}(D)}, \quad|\theta| \gg 1, \quad \theta \in M
$$


In place of the estimate (3.41) one uses the estimate

$$
\|R\|_{L^{\infty}\left(D_{1}\right)} \leq c\left(|\theta|^{-1} \ln |\theta|\right)^{1 / 2}\|q\|_{L^{2}\left(B_{a}\right)}\|R\|_{L^{\infty}\left(D_{1}\right)}+c\left(|\theta|^{-1} \ln |\theta|\right)^{1 / 2}\|q\|_{L^{2}\left(B_{a}\right)}
$$

which implies

$$
\|R\|_{L^{\infty}\left(D_{1}\right)} \leq c\left(|\theta|^{-1} \ln |\theta|\right)^{1 / 2}, \quad q \in Q_{a}, \quad \theta \in M, \quad|\theta| \gg 1
$$

Using (3.50) and the first inequality (3.41) one obtains

$$
\|R\|_{a} \leq c|\theta|^{-1}\|R\|_{L^{\infty}\left(D_{1}\right)}\|q\|_{a}+c|\theta|^{-1}\|q\| \leq c_{1}|\theta|^{-1}
$$

Lemma 12 is proved.

Proof of Lemma 13. Suppose that $w \not \equiv 0$ and (3.8) is false. Then $w$ is orthogonal in $L^{2}\left(S^{2}\right)$ to all functions of the form

$$
\int_{S^{2}} u(x, \alpha) \nu(\alpha) d \alpha,
$$

that is

$$
0=\int_{D} \overline{w(x)} \int_{S^{2}} u(x, \alpha) \nu(\alpha) d \alpha d x \text { for all } \nu \in L^{2}\left(S^{2}\right)
$$

Thus

$$
0=\int_{D} \overline{w(x)} u(x, \alpha) d x \text { for all } \alpha \in S^{2}
$$

It is proved in $[3, \mathrm{p} .46]$ that

$$
G(x, y)=\frac{\exp (i|y|)}{4 \pi|y|} u(x, \alpha)+o\left(|y|^{-1}\right) \text { as }|y| \rightarrow \infty, \quad \frac{y}{|y|}=-\alpha
$$

where $G(x, y)$ is the resolvent kernel for $\ell_{q}: \ell_{q} G=-\delta(x-y)$ in $R^{3}, G$ satisfies the radiation condition, $\ell_{q}$ is defined in (1.1). Therefore (3.53) implies

$$
0=\int_{D} \overline{w(x)} G(x, y) d x:=h(y) \text { for all } y \in D^{\prime}
$$

The function $h(y)$ satisfies the equations

$$
\ell_{q} h=-\bar{w}(x) \text { in } R^{3}
$$

Since $h \in H_{\mathrm{loc}}^{2}$, it follows from (3.55) that

$$
h=h_{N}=0 \text { on } \partial D
$$

Multiply (3.56) by $w(x)$, integrate over $D$ and then by parts, using (3.57) and the equation $\ell_{q} w=0$ in $D$, to get

$$
0=\int_{D}|w|^{2} d x
$$

Therefore $w=0$. This contradiction proves Lemma 13 .

Proof of Lemma 14. Suppose (3.9) is false. Then

$$
\left\|\nu_{\varepsilon}(\alpha)\right\| \leq c \text { for all } \varepsilon \in\left(0, \varepsilon_{0}\right), \quad \varepsilon_{0}>0
$$


Choose a weakly convergent in $L^{2}\left(S^{2}\right)$ subsequence and denote it $\nu_{\varepsilon}(\alpha)$ again, $\varepsilon \rightarrow 0$. Pass to the limit $\varepsilon \rightarrow 0$ in (3.8) with $w=\psi(x, \theta)$ and this subsequence $\nu_{\varepsilon}(\alpha), \nu_{\varepsilon}(\alpha) \rightarrow \nu(\alpha)$ weakly in $L^{2}\left(S^{2}\right)$. The result is

$$
\psi(x, \theta)=\int_{S^{2}} u(x, \alpha) \nu(\alpha) d \alpha \text { in } D, \quad \operatorname{Im} \theta \neq 0
$$

Since both sides in (3.60) solve the elliptic equation

$$
\ell_{q} \psi=0 \text { in } R^{3}
$$

they are identical in $R^{3}$ by the unique continuation property for the solution to $(*)$. This is a contradiction since $\psi(x, \theta)$ grows exponentially as $|x| \rightarrow \infty$ in some directions, while the integral

$$
\left|\int_{S^{2}} u(x, \alpha) \nu(\alpha) d \alpha\right| \leq c\|\nu\|
$$

is bounded. Here we used the well known estimate

$$
\sup _{x \in R^{3}, \alpha \in S^{2}}|u(x, \alpha)| \leq c
$$

Estimate (3.9) is proved. In order to prove (3.10) one deduces from (3.8) with $w=\psi(x, \theta)$ that

$$
\left\|\int_{S^{2}} u(x, \alpha) \nu_{\varepsilon}(\alpha, \theta) d \alpha\right\|_{L^{2}(D)} \geq\|\psi\|_{L^{2}(D)}-\varepsilon \geq c \exp (\kappa d / 2), \quad \kappa=|\operatorname{Im} \theta|
$$

where $d=\operatorname{diam} D$. Assume that for some $\varepsilon>0$ the estimate (3.10) is false. Then there is a sequence $\theta_{n} \in M$, $\left|\theta_{n}\right| \rightarrow \infty$, such that

$$
\left\|\nu_{\varepsilon}\right\| \exp \left(-\kappa_{n} d / 2\right) \rightarrow 0
$$

This contradicts inequality (3.62). Indeed, from (3.62) it follows that

$$
\left.0<c \leq \exp \left(-\kappa_{n} d / 2\right) \| \int_{S^{2}} u(x, \alpha) \nu_{\varepsilon}\left(\alpha, \theta_{n}\right) d \alpha\right)\left\|_{L^{2}(D)} \leq c_{1} \exp \left(-\kappa_{n} d / 2\right)\right\| \nu_{\varepsilon}\left(\alpha, \theta_{n}\right) \|_{L^{2}\left(S^{2}\right)}
$$

where $c_{1}>0$ does not depend on $\varepsilon$ or $\theta_{n}$, it depends on $\max _{x \in R^{3}, \alpha \in S^{2}}|u(x, \alpha)|$ (see (3.61)) and on (meas $D)^{1 / 2}$. Since (3.64) contradicts (3.63), Lemma 14 is proved.

We will use Lemma 17 in the proof of Lemmas 15 and 16. Therefore let us first prove Lemma 17.

Proof of Lemma 17: First, let us prove that $N\left(I+T_{q}\right)=\{0\}$, where $N(A)$ is the null space of a linear operator $A$. It is easy to see that, for $q \in Q_{a}$, the operator $T_{q}$ in (3.17) is a linear compact operator on $L^{2}\left(B_{a}\right)$. Therefore $\left(I+T_{q}\right)^{-1}$ exists and is bounded by the Fredholm alternative if $N\left(I+T_{q}\right)=\{0\}$. Suppose $w+T_{q} w=0$ in $B_{a}$. Define $w(x)$ to be $-T_{q} w$ for any $x \in R^{3}$. Then $w+T_{q} w=0$ in $R^{3}$. Therefore $w$ solves the problem:

$$
\left[\nabla^{2}+1-q(x)\right] w=0 \text { in } R^{3}, \quad|x|\left(\frac{\partial w}{\partial|x|}-i w\right) \rightarrow 0, \quad|x| \rightarrow \infty
$$

It is well known [19] that (3.65) implies $w=0$. Therefore $N\left\{I+T_{q}\right\}=\{0\}$ and the operator $\left(I+T_{q}\right)^{-1}$ exists and is bounded in $L^{2}\left(B_{a}\right)$. Let us prove (3.18). Assume that (3.18) is false. Then there is a sequence $q_{n} \in \mathcal{B}_{C}$ such that

$$
\left\|\left(I+T_{n}\right)^{-1}\right\| \geq n, \quad T_{n}:=T_{q_{n}} .
$$

We prove that this is impossible. Indeed, since $\mathcal{B}_{C}$ is a compact set in $L^{2}\left(B_{a}\right)$, one can select a convergent in $L^{2}\left(B_{a}\right)$ subsequence which we denote again by $q_{n},\left\|q_{n}-q\right\|_{a} \rightarrow 0$ as $n \rightarrow \infty$. One can check that

$$
\left\|T_{q_{1}}-T_{q_{2}}\right\| \leq c\left\|q_{1}-q_{2}\right\|_{a}
$$


Indeed

$$
\left\|T_{q_{1}} f-T_{q_{2}} f\right\|^{2} \leq \frac{1}{(4 \pi)^{2}}\left(\int_{B_{a}} d x \int_{B_{a}} \frac{\left|q_{1}-q_{2}\right|^{2}}{|x-y|^{2}} d y\right)\|f\|^{2} \leq c\left\|q_{1}-q_{2}\right\|_{a}^{2}\|f\|^{2}
$$

from which (3.66) follows with $c=c(a)$. Therefore, if $q_{n} \rightarrow q$ in $L^{2}\left(B_{a}\right)$ then $\left\|T_{n}-T\right\| \rightarrow 0$ as $n \rightarrow \infty$. One has, using bounded invertibility of $I+T$,

$$
\left\|\left(I+T_{n}\right)^{-1}\right\|=\left\|\left(I+T+T_{n}-T\right)^{-1}\right\|=\left\|(I+T)^{-1}\left[I+\left(T_{n}-T\right)(I+T)^{-1}\right]^{-1}\right\| \leq c
$$

Here the inequality holds for all sufficiently large $n$, for example, for such $n$ that $\left\|T_{n}-T\right\|\left\|(I+T)^{-1}\right\|<1$. Since (3.67) contradicts $(*)$, Lemma 17 is proved.

Proof of Lemma 15: Assume that $\theta \in M,|\theta| \gg 1$, is fixed. First, note that one can find $\nu(\alpha)$ such that $\|\nu\|$ is arbitrary large but

$$
\left\|\int_{S^{2}} u(x, \alpha) \nu(\alpha) d \alpha\right\|_{L^{2}(D)} \leq \delta
$$

where $\delta>0$ is an arbitrary small number. Indeed, $u=B u_{0}, u_{0}:=\exp (i \alpha \cdot x)$ and $B=(I+T)^{-1}$ is a bounded linear operator in $L^{2}(D),\|B\|+\left\|B^{-1}\right\| \leq c$ (see Lemma 17). Since $\|h\|=\left\|B^{-1} B h\right\| \leq\left\|B^{-1}\right\|$ $\|B h\|$, one concludes from $(*)$ that

$$
c\left\|\int_{S^{2}} \exp (i \alpha \cdot x) \nu(\alpha) d \alpha\right\|_{L^{2}(D)} \leq \delta
$$

where $c$ does not depend on $\delta$. Conversely, (**) implies $(*)$ (with $c_{1} \delta$ in place of $\delta$ ) since $B$ is a bounded operator. Take $\nu_{\ell}(\alpha)=c_{\ell} Y_{\ell}(\alpha),\|\nu\|=\left|c_{\ell}\right|$. Take $\left|c_{\ell}\right| \rightarrow \infty$ as $\ell \rightarrow \infty$ so that $\left|c_{\ell} j_{\ell}(d)\right| \rightarrow 0$ as $\ell \rightarrow \infty$ where $d=$ diam $D$. Such a choice of $c_{\ell}$ is possible since $\left|j_{\ell}(b)\right| \rightarrow 0$ as $\ell \rightarrow \infty$ (see (3.19)). So, $\left\|\nu_{\ell}(\alpha)\right\| \rightarrow \infty$, and

$$
\left\|\int_{S^{2}} u(x, \alpha) \nu_{\ell}(\alpha) d \alpha\right\|_{L^{2}(D)} \leq c\left\|\int_{S^{2}} \exp (i \alpha \cdot x) c_{\ell} Y_{\ell}(\alpha) d \alpha\right\|_{L^{2}(D)}=c^{\prime}\left|c_{\ell}\right|\left\|j_{\ell}(r)\right\|_{L^{2}(D)} \rightarrow 0 \text { as } \ell \rightarrow \infty
$$

The claim is proved. That is why we are looking for $\nu_{\varepsilon}(\alpha)$ which satisfies (3.11) and has minimal norm.

Let us note that $\psi=B \varphi$, where $\varphi$ solves the equation

$$
\nabla^{2} \varphi+\varphi=0 \text { in } R^{3}
$$

Indeed, since $\varphi=B^{-1} \psi=(I+T) \psi$, one has $\left(\nabla^{2}+1\right) \varphi=\left(\nabla^{2}+1\right)(I+T) \psi=q \psi-q \psi=0$ as claimed. Inequality (3.11) implies, as in the passage from $(*)$ to $(* *)$, that

$$
\left\|\varphi-\int_{S^{2}} \exp (i \alpha \cdot x) \nu_{\varepsilon}(\alpha) d \alpha\right\| \leq c \varepsilon:=\varepsilon_{1}, \quad b>a_{1}>a \sqrt{2}
$$

where $c$ does not depend on $\varepsilon$ and $\theta$, the norm $\|\cdot\|$ in this argument is equivalent to the norm $\|\cdot\|_{L^{2}\left(B_{b}\right)}$, and

$$
\varphi=\psi+T \psi=\psi+\int_{B_{a}} \frac{\exp (i|x-y|)}{4 \pi|x-y|} q(y) \psi(y, \theta) d y .
$$

Since $b>a$ and $|\theta| \gg 1,\|\varphi\|_{b}$ is of order of $\|\psi\|_{b}$ which, in turn, is of order of $\|\exp (i \theta \cdot x)\|_{b}$ as $|\theta| \rightarrow \infty$. Also, $\|\varphi\|_{b},\|\psi\|_{b}$ and $\|\exp (i \theta \cdot x)\|_{b}$ are of order $\|\exp (i \theta \cdot x)\|$ as $|\theta| \rightarrow \infty, \theta \in M$, where $\|\cdot\|$ is defined in (2.14). Since $\varphi$ solves (3.68) one can write

$$
\varphi(x)=\sum_{\ell=0}^{\infty} a_{\ell} j_{\ell}(r) Y_{\ell}\left(\alpha^{\prime}\right), \quad r=|x|, \quad \alpha^{\prime}=x / r .
$$


Also

$$
\exp (i \alpha \cdot x)=\sum_{\ell=0}^{\infty} 4 \pi i^{\ell} j_{\ell}(r) Y_{\ell}\left(\alpha^{\prime}\right) \overline{Y_{\ell}(\alpha)}, \quad r=|x|, \quad \alpha^{\prime}=x / r
$$

Thus (3.69) can be written as

$$
\sum_{\ell=0}^{\infty}\left|a_{\ell}-4 \pi i^{\ell} \nu_{\varepsilon \ell}\right|^{2} b_{\ell}^{2} \leq \varepsilon_{1}^{2}, \quad b_{\ell}^{2}:=\int_{0}^{b} r^{2}\left|j_{\ell}(r)\right|^{2} d r \quad \nu_{\varepsilon \ell}:=\left(\nu_{\varepsilon}, Y_{\ell}\right)_{L^{2}\left(S^{2}\right)}
$$

One can write an equation similar to $(3.17)$ for $\psi$ :

$$
(I+\Gamma) \psi=\exp (i \theta \cdot x), \quad \Gamma \psi:=\Gamma_{q} \psi:=\int_{B_{a}} G(x, y, \theta) q \psi d y .
$$

Here $G$ solves the equation

$$
\begin{gathered}
\left(\nabla^{2}+1\right) G=-\delta(x), \quad G=\exp (i \theta \cdot x) g, \\
L g:=\left(\nabla^{2}+2 i \theta \cdot \nabla\right) g=-\delta(x), \quad g=\frac{1}{(2 \pi)^{3}} \int_{R^{3}} \frac{\exp (i \lambda \cdot x)}{\lambda^{2}+2 \lambda \cdot \theta} d \lambda .
\end{gathered}
$$

The operator $(I+\Gamma)^{-1}$ exists and is bounded in $L^{2}\left(B_{a}\right)$ if $|\theta| \gg 1$. To prove this, it is sufficient, as in the proof of Lemma 17, to prove that $N(I+\Gamma)=\{0\}$. Suppose $w+\Gamma w=0$ in $B_{a}$. Then $w$ solves equation $(3.65)$ and satisfies the following condition at infinity: $w=\exp (i \theta \cdot x) v$, where

$$
v=L^{-1} q v \text { in } R^{3} .
$$

and $L^{-1}$ is defined in (3.36). The relation (3.77) plays the role of the condition at infinity. Using estimate (3.40) one derives from (3.77) restricted to $B_{a}$ that $v=0$ provided that $|\theta| \gg 1$, namely, $|\theta|$ is so large that $\left\|L^{-1} q\right\|_{L^{2}\left(B_{a}\right) \rightarrow L^{2}\left(B_{a}\right)}<1$. Thus $v=0, w=0$, and the operator $(I+\Gamma)^{-1}$ is bounded in $L^{2}\left(B_{a}\right)$. Since $\Gamma$ is a compact linear integral operator in $L^{2}\left(B_{a}\right)$ and $I+\Gamma$ is injective, one can write $(I+\Gamma)^{-1}=I+\Gamma_{1}$ where $\Gamma_{1}$ is a compact linear integral operator in $L^{2}\left(B_{a}\right)$. Therefore

$$
\psi=\exp (i \theta \cdot x)+\Gamma_{1} \exp (i \theta \cdot x)=\exp (i \theta \cdot x)+\int_{B_{a}} \Gamma_{1}(x, y) \exp (i \theta \cdot y) d y,
$$

and from (3.70) one obtains

$$
\varphi=\exp (i \theta \cdot x)+\Gamma_{1} \exp (i \theta \cdot x)+T \exp (i \theta \cdot x)+T \Gamma_{1} \exp (i \theta \cdot x) .
$$

It follows from (3.79) that $\exp (i \theta \cdot x)$ is the main term of $\varphi$ as $|\theta| \rightarrow \infty$ in the region $|x|>a$. Since $\varphi=\psi+T \psi$ and $|\psi| \leq c \exp (\kappa r)$ one has $|\varphi| \leq c \exp (\kappa r), r>a$. Thus

$$
\|\varphi\|_{L^{2}\left(S^{2}\right)}^{2}=\sum_{\ell=0}^{\infty}\left|a_{\ell}\right|^{2} j_{\ell}^{2}(r)<c \exp (2 \kappa r), \quad r>a
$$

and

$$
\left|a_{\ell}\right|<c \exp (\kappa r)\left|j_{\ell}(r)\right|^{-1}, \quad r>a .
$$

Define

$$
\nu_{\varepsilon \ell}:=\left(4 \pi i^{\ell}\right)^{-1} a_{\ell} \quad \text { for } l \leq N, \quad \nu_{\varepsilon \ell}:=0 \text { for } \ell>N .
$$


Then, using (3.80) and (2.9), one gets

$$
\begin{gathered}
\left\|\nu_{\varepsilon}\right\|^{2}=\sum_{\ell=0}^{\infty}\left|\nu_{\varepsilon \ell}\right|^{2}=\sum_{\ell=0}^{N}\left|\nu_{\varepsilon \ell}\right|^{2} \leq c \exp (2 \kappa r) \sum_{\ell=0}^{N} \frac{2 \ell+1}{\left|j_{\ell}(r)\right|^{2}} \leq \\
\leq c \exp (2 \kappa r) \sum_{\ell=0}^{N}\left(\frac{2 \ell+1}{e r}\right)^{2 \ell+1}(2 \ell+1)^{2} \leq c \exp (2 \kappa r)\left(\frac{2 N+1}{e r}\right)^{2 N+1} N^{3},
\end{gathered}
$$

where $r>0$ is such that $j_{\ell}(r) \neq 0$ for all $\ell \geq 0$. Let us fix $r>b$ and estimate $b_{\ell}$ in (3.73), for large $\ell$, using $(3.19)$ :

$$
b_{\ell}^{2} \leq \int_{0}^{b} r\left(\frac{e r}{2 \ell+1}\right)^{2 \ell+1} \frac{1}{2 \ell+1} d r \leq c \frac{e^{2 \ell+1} b^{2 \ell+3}}{(2 \ell+1)^{2 \ell+2}(2 \ell+3)} \leq c \frac{(e b)^{2 \ell}}{(2 \ell)^{2 \ell+3}} \leq c\left(\frac{e b}{2 \ell+1}\right)^{2 \ell+1} \frac{1}{\ell^{2}}
$$

From (3.81), (3.82) and (3.84) one concludes that (3.73) holds if

$$
\begin{aligned}
& c \sum_{\ell=N+1}^{\infty} \exp (2 \kappa r)\left[\left(\frac{e r}{2 \ell+1}\right)^{2 \ell+1} \frac{1}{2 \ell+1}\right]^{-1}\left(\frac{e b}{2 \ell+1}\right)^{2 \ell+1} \frac{1}{\ell^{2}} \leq \\
& \leq c \exp (2 \kappa r) \sum_{\ell=N+1}^{\infty}\left(\frac{b}{r}\right)^{2 \ell+1} \leq c \exp (2 \kappa r)\left(\frac{b}{r}\right)^{2 N} \leq \varepsilon_{1}^{2}, \quad r>b
\end{aligned}
$$

Write (3.85) as

$$
\exp (\kappa r)\left(\frac{b}{r}\right)^{N} \leq \varepsilon_{2}, \quad \varepsilon_{2}=\varepsilon_{1} / \sqrt{c}:=c_{2} \varepsilon, \quad N \gg 1 .
$$

Since $r>b$ is arbitrary, one can minimize the left-hand side of (3.86) in $r$ and get

$$
\inf _{r>b}\left[\exp (\kappa r)\left(\frac{b}{r}\right)^{N}\right]=\exp (N)\left(\frac{b \kappa}{N}\right)^{N}
$$

where the infimum is attained at $r=N / \kappa$. Consider the equality in (3.86) with this $r$ :

$$
\left(\frac{e b \kappa}{N}\right)^{N}=\varepsilon_{2}, \quad \varepsilon_{2} \rightarrow 0
$$

Let us solve (3.87) for $N$ asymptotically as $\varepsilon_{2} \rightarrow 0$, for a fixed $\kappa$. Write (3.87) as

$$
\ln \ln \varepsilon_{2}^{-1}=\ln N+\ln \ln N-\frac{\ln (e b \kappa)}{\ln N}+o\left(\frac{1}{\ln N}\right)=(\ln N)\left[1+O\left(\frac{\ln \ln N}{\ln N}\right)\right] .
$$

Therefore

$$
\ln N=\left(\ln \ln \varepsilon_{2}^{-1}\right)[1+o(1)], \quad \varepsilon_{2} \rightarrow 0
$$

Thus

$$
N=\exp \left\{\left(\ln \ln \varepsilon_{2}^{-1}\right)[1+o(1)]\right\}, \quad \varepsilon_{2}=c_{2} \varepsilon, \quad \varepsilon \rightarrow 0 .
$$

With $N=N(\varepsilon)$ given by (3.90) formula (3.83) yields an upper estimate for $\left\|\nu_{\varepsilon}\right\|$ and therefore for $j(\varepsilon, \theta)$ for arbitrary large fixed $\theta \in M$ and $\varepsilon \rightarrow 0$ :

$$
j(\varepsilon, \theta) \leq c \exp (\kappa r)\left(\frac{2 N+1}{e r}\right)^{N} N^{2} .
$$


where $N$ is given by (3.90). Minimize the right-hand size of (3.91) in $r, r>b$. As above, the minimum is attained at $r=N / \kappa$ and (3.91) with this $r$ yields

$$
j(\varepsilon, \theta) \leq c\left(\frac{2 N+1}{N} \kappa\right)^{N} N^{2} \leq c(2 \kappa)^{N} N^{2}
$$

Since $N$ in (3.90) can be written as $n(\varepsilon)$ in (3.13), Lemma 15 is proved.

Proof of Lemma 16: If $\varepsilon=\exp (-\kappa b) / \kappa, \kappa=|\operatorname{Im} \theta|$, then (3.87) takes the form

$$
\left(\frac{e b \kappa}{N}\right)^{N}=c_{2} \frac{\exp (-\kappa b)}{\kappa}
$$

Let $N=N(\kappa)$ solve (3.93) asymptotically as $\kappa \rightarrow+\infty$. Denote $\nu_{\varepsilon}(\alpha, \theta)$ corresponding to $\varepsilon=\varepsilon(\kappa):=$ $\kappa^{-1} \exp (-\kappa b)$ by $\nu_{\kappa}$. The Fourier coefficients of $\nu_{\kappa}$ are given by (3.82) with $N=N(\kappa)$. From (3.83) one derives

$$
j(\varepsilon(\kappa), \theta) \leq c \frac{\exp (2 \kappa r)}{r^{2 N(\kappa)+1}}\left(\frac{2 N(\kappa)+1}{e}\right)^{2 N(\kappa)+1} N^{3}(\kappa), \quad r>b .
$$

Minimizing in $r>b$ the right-hand side of (3.94) one obtains, at $r=(2 N(\kappa)+1) /(2 \kappa)$,

$$
\begin{aligned}
& j(\varepsilon, \theta) \leq c \frac{\exp [2 N(\kappa)+1]}{\left(\frac{2 N(\kappa)+1}{2 \kappa}\right)^{2 N(\kappa)+1}}\left(\frac{2 N(\kappa)+1}{e}\right)^{2 N(\kappa)+1} N^{3}(\kappa)= \\
= & c \exp [2 N(\kappa)+1]\left(\frac{2 \kappa}{e}\right)^{2 N(\kappa)+1} N^{3}(\kappa)=c N^{3}(\kappa)(2 \kappa)^{2 N(\kappa)+1} .
\end{aligned}
$$

Estimate (3.16) is proved. To prove $\left(3.16^{\prime}\right)$, note that if $N(\kappa)<e b \kappa$ or $N(\kappa)>e^{2} b \kappa$ then (3.15) cannot hold as $\kappa \rightarrow \infty$. Indeed, as $\kappa \rightarrow \infty$ then $(e b \kappa / N)^{N}>1>c_{2} \exp (-\kappa b) / \kappa$ for $N(\kappa)<e b \kappa$ and

$$
\left(\frac{e b \kappa}{N}\right)^{N}<\left(\frac{1}{e}\right)^{N}<\exp \left(-e^{2} b \kappa\right)<c_{2} \kappa^{-1} \exp (-\kappa b) \text { for } N(\kappa)>e^{2} b \kappa .
$$

Lemma 16 is proved.

\section{Proofs of Lemmas 1-9}

$\square$

Proof of Lemma 1: One has, using (3.72) and the orthonormality of the spherical harmonics

$$
\begin{aligned}
& \left|A_{\ell m}(\alpha)\right|=\frac{1}{4 \pi}\left|\int_{B_{a}} q(x) u(x, \alpha) \int_{S^{2}} \exp (-i \beta \cdot x) \overline{Y_{\ell m}(\beta)} d \beta d x\right| \leq \\
& \leq c \int_{B_{a}}\left|q(x) j_{\ell}(r) Y_{\ell m}\left(\alpha^{\prime}\right)\right| d x, \quad r=|x|, \quad \alpha^{\prime}=x / r
\end{aligned}
$$

and $c$ is the constant from (3.61). From (4.1) using (3.19) one obtains

$$
\left|A_{\ell m}(\alpha)\right| \leq c\|q\|_{a}\left(\int_{0}^{a} r^{2}\left|j_{\ell}(r)\right|^{2} d r\right)^{1 / 2} \leq c_{1} a\left(\frac{e a}{2 \ell+1}\right)^{(2 \ell+1) / 2} \frac{1}{2 \ell+1}
$$

Lemma 1 is proved.

Proof of Lemma 2: Using the formula

$$
\int_{S^{2}} \exp (i \theta \cdot \alpha r) Y_{\ell}(\alpha) d \alpha=4 \pi i^{\ell} j_{\ell}(r) Y_{\ell}(\theta), \quad \theta \in M
$$


where $r>0$ is arbitrary, one applies the Cauchy inequality to the integral in (4.3) and obtains

$$
\left|Y_{\ell}(\theta)\right| \leq \frac{\exp (\kappa r)}{4 \pi\left|j_{\ell}(r)\right|}\left\|Y_{\ell}(\alpha)\right\|\left(\int_{S^{2}} d \alpha\right)^{1 / 2}=\frac{\exp (\kappa r)}{\sqrt{4 \pi}\left|j_{\ell}(r)\right|}
$$

Lemma 2 is proved.

Proof of Lemma 3: The function $w$, defined by the right-hand side of (2.15), solves the Helmholtz equation

$$
\left(\nabla^{2}+1\right) w=0 \text { for } r>a
$$

and has the asymptotics

$$
w=\exp (i \alpha \cdot x)+A\left(\alpha^{\prime}, \alpha\right) r^{-1} \exp (i r)+o\left(r^{-1}\right), \quad r \rightarrow \infty, \quad \alpha^{\prime}=x / r
$$

Therefore the function $v:=w-u(x, \alpha)$, where $u(x, \alpha)$ is the scattering solution, solves equation (4.5) in the region $r>a$ and

$$
v=o\left(r^{-1}\right), \quad r \rightarrow \infty
$$

By the Rellich-type uniqueness lemma (see e.g. [3, p. 24]) one has $v=0$ for $r>a$. Lemma 3 is proved. $\quad \square$ Remark: Lemma 3 is well known.

Proof of Lemma 4: One has, with $\varphi:=\int_{S^{2}} u(x, \alpha) \nu(\alpha) d \alpha$,

$$
\begin{aligned}
& \|\rho(\nu)\|:=\left\|\exp (-i \theta \cdot x) \int_{S^{2}} u(x, \alpha) \nu(\alpha) d \alpha-1\right\|= \\
= & \|\exp (-i \theta \cdot x)[\varphi-\exp (i \theta \cdot x)-\exp (i \theta \cdot x) R(x, \theta)+\exp (i \theta \cdot x) R(x, \theta)]\| \leq \\
& \|\exp (-i \theta \cdot x)[\varphi-\psi]\|+\|R(x, \theta)\|
\end{aligned}
$$

By Lemma 13, with $w=\psi$ and $\psi$ as in Lemma 12, one can find $\nu=\nu(\alpha, \theta)$ such that

$$
\|\varphi-\psi\| \leq \frac{\exp (-\kappa b)}{\kappa}, \quad \kappa:=|\operatorname{Im} \theta|
$$

With this choice of $\nu$ one has

$$
\|\exp (-i \theta \cdot x)[\varphi-\psi]\| \leq \exp (\kappa b)\|\varphi-\psi\| \leq \kappa^{-1}
$$

Since $\theta \in M$ one has $|\theta| / \kappa \rightarrow \sqrt{2}$ as $\theta \rightarrow \infty$. From this, (4.7), (4.8) and (4.9) estimate (2.16) follows. Lemma 4 is proved.

Proof of Lemma 5: Let us choose the coordinate system so that $\lambda=t e_{3}$, where $t>0$ and $e_{j}, j=1,2,3$, is an orthonormal basis of $R^{3}$. Let $\theta^{\prime}:=\frac{1}{2} t e_{3}+v, \theta:=-\frac{1}{2} t e_{3}+v$, where $v \cdot v=1-t^{2} / 4, v_{3}=0,|v| \rightarrow \infty, v \in C^{2}$. Clearly there are infinitely many such $v$. If $t \neq 1$, then one can choose $\theta^{\prime}$ and $\theta$ in $M^{\prime}$ in the above proof. Lemma 5 is proved.

The reader can also consult [4] for another proof.

The proof of Lemma 6 is given in Section V.

Let us prove the remaining lemmas.

Proof of Lemma 7: One has

$$
\begin{aligned}
& \left\|\rho_{\delta}(\nu)\right\|:=\left\|\exp (-i \theta \cdot x) \int_{S^{2}} u_{\delta}(x, \alpha) \nu(\alpha) d \alpha-1\right\| \leq \\
& \leq\left\|\exp (-i \theta \cdot x) \int_{S^{2}} u(x, \alpha) \nu(\alpha) d \alpha-1\right\|+\left\|\exp (-i \theta \cdot x) \int_{S^{2}}\left[u_{\delta}(x, \alpha)-u(x, \alpha)\right] \nu(\alpha) d \alpha\right\| \leq \\
& \leq\|\rho(\nu)\|+c \exp (\kappa b) a(\nu) \sup _{\alpha \in S^{2}}\left\|u_{\delta}(x, \alpha)-u(x, \alpha)\right\|
\end{aligned}
$$


Let us prove that

$$
\sup _{\alpha \in S^{2}}\left\|u_{\delta}(x, \alpha)-u(x, \alpha)\right\| \leq c \mu(\delta)
$$

where $\mu(\delta)$ is defined in (2.28) and $N(\delta)$ is defined in (2.24). One has, with $N=N(\delta), r=|x|$ and $\alpha^{\prime}=x / r$,

$$
\left\|u_{\delta}(x, \alpha)-u(x, \alpha)\right\| \leq\left\|\sum_{\ell=0}^{N}\left[A_{\delta \ell}(\alpha)-A_{\ell}(\alpha)\right] Y_{\ell}\left(\alpha^{\prime}\right) h_{\ell}(r)\right\|+\left\|\sum_{\ell=N+1}^{\infty} A_{\ell}(\alpha) Y_{\ell}\left(\alpha^{\prime}\right) h_{\ell}(r)\right\|:=I_{1}+I_{2}
$$

Using Parseval's equality and the assumption (1.3) one gets

$$
I_{1}^{2}=\max _{\alpha \in S^{2}} \sum_{\ell=0}^{N}\left|A_{\delta \ell}(\alpha)-A_{\ell}(\alpha)\right|^{2} \int_{a_{1}}^{b} r^{2}\left|h_{\ell}(r)\right|^{2} d r \leq 4 \pi \delta^{2} \max _{0 \leq \ell \leq N} \int_{a_{1}}^{b} r^{2}\left|h_{\ell}(r)\right|^{2} d r .
$$

Furthermore

$$
I_{2}^{2} \leq \max _{\alpha \in S^{2}} \sum_{\ell=N+1}^{\infty}\left|A_{\ell}(\alpha)\right|^{2} \int_{a_{1}}^{b} r^{2}\left|h_{\ell}(r)\right|^{2} d r
$$

Define

$$
H_{\ell}:=\int_{a_{1}}^{b} r^{2}\left|h_{\ell}(r)\right|^{2} d r
$$

The following formula is known:

$$
\left|H_{\ell}^{(1)}(r)\right|^{2}=\frac{4}{\pi^{2}} \int_{0}^{\infty} K_{0}(2 r \operatorname{sh}(t))\left(e^{2 \ell t}+e^{-2 \ell t}\right) d t,
$$

where $H_{\ell}^{(1)}(r)$ is the Hankel function and $K_{0}(r)$ is the Macdonald function. It follows from this formula that $\left|H_{\ell}^{(1)}(r)\right|$ is a monotonically increasing function of $\ell$. It is known that $r\left|H_{\ell}^{(1)}(r)\right|^{2}$ is a monotonically decreasing function of $r>0$ if $\ell>\frac{1}{2}$. One can prove (see [5]) that

$$
H_{\ell} \leq c\left(\frac{2 \ell+1}{e a_{2}}\right)^{2 \ell} \ell^{1 / 2}, \quad a_{2}=\frac{a_{1}}{\sqrt{2}}
$$

In fact, a stronger estimate holds:

$$
H_{\ell} \leq c a_{1}^{2}[(2 \ell+1) \ell]^{-1}\left(\frac{2 \ell+1}{e a_{1}}\right)^{2 \ell+1} .
$$

This follows from the known asymptotics of Hankel's functions: $h_{\ell}(r) \sim-i(r \ell)^{-0.5}\left(\frac{2 \ell+1}{e r}\right)^{\ell+0.5}$ as $\ell \rightarrow \infty$.

From (4.13)-(4.16') and (2.7) one gets

$$
I_{1}+I_{2} \leq c\left[\delta\left(\frac{2 N+1}{e a_{1}}\right)^{N+1}+\left(\frac{a}{a_{1}}\right)^{N}\right]
$$

Minimizing with respect to $N$ the expression in brackets in (4.17) for a small fixed $\delta>0$ one obtains that the minimum is $c \mu_{1}(\delta)$ with $\mu_{1}(\delta)$ defined in (2.28) and $N=N(\delta)$ defined in (2.24) (cf. [5]). Inequality (2.30) is proved. Inequality $(2.31)$ can be proved similarly. Lemma 7 is proved.

Proof of Lemma 8: Use (2.30) to conclude that (2.32) holds if

$$
\|\rho(\nu)\|+a(\nu) \exp (\kappa b) \mu(\delta) \leq c|\theta|^{-1}
$$


Using Lemma 13 with $\varepsilon=|\theta|^{-1} \exp (\kappa b), \kappa=|\operatorname{Im} \theta|$ and $D=B_{b}$, choose $\nu=\nu(\alpha, \theta)$ such that

$$
\|\varphi-\psi\| \leq|\theta|^{-1} \exp (-\kappa b)
$$

where

$$
\varphi:=\int_{S^{2}} u(x, \alpha) \nu(\alpha, \theta) d \alpha
$$

Then, using (3.6), (3.7) and (4.19), one gets

$$
\begin{aligned}
& \|\rho(\nu)\|=\|\exp (-i \theta \cdot x) \varphi-1\| \leq\|\exp (-i \theta \cdot x)[\varphi-\psi]\|+\|R\| \leq \\
& \leq \exp (\kappa b)\|\varphi-\psi\|+c|\theta|^{-1} \leq|\theta|^{-1}(c+1)
\end{aligned}
$$

Choose $\theta=\theta(\delta)$ such that

$$
|\theta| a(\nu(\alpha, \theta)) \exp (\kappa b)=1 / \mu(\delta)
$$

Note that $\mu^{-1}(\delta) \rightarrow \infty$ as $\delta \rightarrow 0$. We claim that

$$
a(\nu(\alpha, \theta)) \rightarrow \infty \text { as }|\theta| \rightarrow \infty, \quad \theta \in M
$$

From (4.23) it follows that equation (4.22) has a solution $\theta(\delta)$ such that

$$
|\theta(\delta)| \rightarrow \infty \text { as } \delta \rightarrow 0
$$

To finish the proof, let us check that (4.23) holds. Assume the contrary, that is

$$
a(\nu(\alpha, \theta)) \leq c^{\prime}
$$

where $c^{\prime}$ does not depend on $\theta$. Using (4.25) choose a weakly convergent in $L^{2}\left(S^{2}\right)$ subsequence which is denoted by $\nu\left(\alpha, \theta_{n}\right):=\nu_{n}$,

$$
\nu\left(\alpha, \theta_{n}\right) \rightarrow \nu(\alpha) \text { weakly in } L^{2}\left(S^{2}\right) \text { as }\left|\theta_{n}\right| \rightarrow \infty
$$

Pass to the limit in (4.19) to get

$$
\left\|\psi\left(x, \theta_{n}\right)-\varphi\left(x, \theta_{n}\right)\right\| \rightarrow 0, \quad n \rightarrow \infty
$$

where

$$
\varphi\left(x, \theta_{n}\right):=\int_{S^{2}} u(x, \alpha) \nu\left(\alpha, \theta_{n}\right) d \alpha
$$

This is a contradiction since $\|\varphi\| \leq c\left\|\nu_{n}\right\|(4 \pi)^{1 / 2} \leq c c^{\prime}(4 \pi)^{1 / 2} \leq c_{1}$, while $\left\|\psi\left(x, \theta_{n}\right)\right\| \rightarrow \infty$ as $n \rightarrow \infty$. In this estimate $c$ is the constant from (3.61). Lemma 8 is proved.

Remark: The rate of growth of $a(\nu(\alpha, \theta))$ as $|\theta| \rightarrow \infty, \theta \in M$, can be estimated from below by formula (3.10) and from above, for some choice of $\nu(\alpha, \theta)$, by (3.16)-(3.16" $)$. This allows one to get the following lower estimate of $\Theta(\delta)$ which gives a refinement of $(2.33)$ :

$$
\Theta(\delta) \geq|\theta(\delta)| \geq c \frac{|\ln \delta|}{(\ln |\ln \delta|)^{2}}
$$

To prove (4.29) let us solve (4.22), asymptotically as $\delta \rightarrow 0$, for $|\theta(\delta)|$ using the upper estimate $\left(3.16^{\prime \prime}\right)$ for $a(\nu, \theta)$. Equation (4.22) becomes

$$
|\theta| \exp (\kappa b) \kappa^{4}(2 \kappa)^{20 b \kappa}=1 / \mu(\delta)
$$

Note that in order to obtain a lower estimate for $|\theta(\delta)|$ one has to use an upper estimate for $a(\nu)$ in $(4.22)$. Since $|\theta| / \kappa \rightarrow \sqrt{2}$ as $|\theta| \rightarrow \infty, \theta \in M$, one can write (4.30) as

$$
\exp [\kappa b+5 \ln \kappa+20 b \kappa \ln \kappa+20 b \kappa \ln 2]=\exp [\gamma N(\delta)]
$$


where $N(\delta)$ is given in (2.24). Note that the main term of the asymptotic solution to (4.31) will be the same for the equation (4.31) with $c \exp [\gamma N(\delta)]$ in place of $\exp [\gamma N(\delta)], c=$ const. From (4.31) one derives

$$
20 b \kappa(\ln \kappa)\left[1+\left(\frac{1}{\ln \kappa}\right)\right]=\gamma N(\delta)
$$

Thus

$$
\kappa \ln \kappa=\gamma_{1} N(\delta)[1+o(1)], \quad \delta \rightarrow 0, \quad \gamma_{1}:=\frac{\gamma}{20 b}
$$

Taking $\ln$ of (4.33) and denoting $\gamma_{1} N(\delta):=n$ yields

$$
\ln \kappa+\ln \ln \kappa=\ln n+o(1)
$$

Let us look for the asymptotic solution to (4.34) of the form

$$
\kappa=\frac{n}{\ln n}(1+t), \quad t=o(1) \text { as } n \rightarrow \infty
$$

Substitute (4.35) into (4.34) to get

$$
\ln n-\ln \ln n+t+O\left(t^{2}\right)+\ln \left\{(\ln n)\left[1-\frac{\ln \ln n+t+O\left(t^{2}\right)}{\ln n}\right]\right\}=\ln n+o(1)
$$

Thus

$$
t+O\left(t^{2}\right)-\frac{\ln \ln n+t+O\left(t^{2}\right)}{\ln n}=o(1)
$$

Equation (4.36) implies that $t=o(1)$ as $n \rightarrow \infty$. Therefore formula (4.35) gives an asymptotic solution to equation (4.33) as $\delta \rightarrow 0$. Let us write this solution for references in terms of $|\theta(\delta)|=\sqrt{2} \kappa(\delta)[1+o(1)]$ as $\delta \rightarrow 0$

$$
|\theta(\delta)|=\frac{\sqrt{2} \gamma_{1} N(\delta)}{\ln \left[\gamma_{1} N(\delta)\right]}[1+o(1)], \quad \delta \rightarrow 0, \quad \gamma_{1}=\frac{\gamma}{20 b}
$$

where $N(\delta)=|\ln \delta| / \ln |\ln \delta|$. Therefore

$$
|\theta(\delta)|=\frac{\sqrt{2} \gamma_{1}|\ln \delta|}{\ln |\ln \delta|\left[\ln \gamma_{1}+\ln |\ln \delta|-\ln \ln |\ln \delta|\right]}[1+o(1)]=\frac{\sqrt{2} \gamma_{1}|\ln \delta|}{(\ln |\ln \delta|)^{2}}[1+o(1)], \quad \delta \rightarrow 0
$$

Let us formulate the result:

Lemma $8^{\prime}$ : One has

$$
\Theta(\delta) \geq|\theta(\delta)|=\frac{\sqrt{2} \gamma_{1}|\ln \delta|}{(\ln |\ln \delta|)^{2}}[1+o(1)] \text { as } \delta \rightarrow 0
$$

where

$$
\gamma_{1}=\frac{\ln \left[a_{1} / a\right]}{20 b}>0
$$

Proof of Lemma 9: The starting point is the standard resolvent identity

$$
G_{1}(x, y)-G_{2}(x, y)=-\int_{B_{a}} G_{1}(x, z) p(z) G_{2}(z, y) d z
$$

where $p:=q_{1}-q_{2}$ and $G_{j}$ is the resolvent kernel of the operator $\ell_{j}:=\nabla^{2}+1-q_{j}(x)$. Let $|y| \rightarrow \infty, y /|y|=\beta$ in (4.41). Using (3.54) one gets

$$
u_{1}(x,-\beta)-u_{2}(x,-\beta)=-\int_{B_{a}} G_{1}(x, z) p(z) u_{2}(z,-\beta) d z
$$


Let $|x| \rightarrow \infty, x /|x|=-\alpha$ in (4.42) and use (1.2) and (3.54) again to get

$$
A_{1}(-\alpha,-\beta)-A_{2}(-\alpha,-\beta)=\frac{-1}{4 \pi} \int_{B_{a}} p(z) u_{1}(z, \alpha) u_{2}(z,-\beta) d z
$$

By the well known reciprocity property $A_{j}(-\alpha,-\beta)=A_{j}(\beta, \alpha)$, so (4.43) is identical with (2.40). The original proof of Lemma 9, given in [9], was a little longer.

\section{Proof of Lemma 6}

This proof requires some preparation. Consider the equation

$$
L \rho:=\left(\nabla^{2}+2 i \theta \cdot \nabla\right) \rho=v \text { in } R^{3}, \quad \rho \in C_{0}^{2}\left(B_{r}\right), \quad \theta \in M
$$

Let

$$
\begin{gathered}
D_{j}:=-i \frac{\partial}{\partial x_{j}}, \quad D=-i \nabla, \quad \partial_{j}=\frac{\partial}{\partial x_{j}} \\
P(\xi):=\xi^{2}+2 \beta \cdot \xi, \quad \beta:=h \theta \quad h:=|\theta|^{-1} \\
\beta \cdot \beta=h^{2}, \quad|\beta|=1 \\
N:=\left\{\xi: P(\xi)=0, \quad \xi \in R^{3}\right\} \\
N_{h}:=\left\{\xi: \xi \in R^{3}, \quad \operatorname{dist}(\xi, N) \leq h\right\} \\
N_{h}^{\prime}:=R^{3} \backslash N_{h}
\end{gathered}
$$

Let $\beta=m+i \mu, m, \mu \in R^{3}$. Then

$$
N=\left\{\xi:|\xi+m|=|m|, \quad \mu \cdot \xi=0, \quad \xi \in R^{3}\right\}
$$

Thus $N$ is a circle. Let $P(\xi)=P_{1}(\xi)+i P_{2}(\xi)$, where $P_{1}(\xi):=\operatorname{Re} P(\xi)$. Note that

$$
d P_{1}(\xi) \neq 0 \text { for } \xi \in N
$$

where $d P_{1}$ is the differential of $P_{1}$. Equation (5.1) can be written as

$$
P(h D) \rho:=\left[(h D)^{2}+2 \beta \cdot h D\right] \rho=-h^{2} v
$$

Define

$$
\begin{gathered}
F_{h} u:=\hat{u}(\xi):=(2 \pi h)^{-3 / 2} \int_{R^{3}} u(x) \exp \left(-i \xi \cdot x h^{-1}\right) d x \\
u(x)=(2 \pi h)^{-3 / 2} \int_{R^{3}} \hat{u}(\xi) \exp \left(i \xi \cdot x h^{-1}\right) d \xi \\
i h \partial_{\xi_{j}} \hat{u}(\xi)=\widehat{x_{j} u} \\
F_{h}\left\{-i h \partial_{j} u(x)\right\}=\xi_{j} \hat{u}(\xi)
\end{gathered}
$$

Let us denote in this section

$$
\|\rho\|:=\|\rho\|_{L^{2}\left(R^{3}\right)}
$$




$$
\begin{gathered}
\|\rho\|_{b_{1}, b_{2}}:=\|\rho\|_{L^{2}\left(B_{b_{2}} \backslash B_{b_{1}}\right)}, \quad 0<b_{1}<b_{2} \\
\|g\langle h D\rangle \rho\|:=\left\|g\left(\sqrt{1+\xi^{2}}\right) \hat{\rho}(\xi)\right\|
\end{gathered}
$$

First, we need

Lemma 18: Any solution $\rho \in C_{0}^{2}\left(B_{r}\right)$ to (5.10) satisfies the inequality

$$
h\left\|\langle h D\rangle^{2} \rho\right\| \leq c\|P(h D) \rho\| \quad \forall h \in\left(0, h_{0}\right)
$$

where $h_{0}>0$ is a fixed sufficiently small number.

Proof: Inequality (5.18) can be written as

$$
h\left\|\left(1+\xi^{2}\right) \hat{\rho}\right\| \leq c\|P(\xi) \hat{\rho}\|
$$

where the definition (5.17) and Parseval's equality are used.

Let $\xi \in N_{h}^{\prime}$. Then

$$
h\left(1+\xi^{2}\right) \leq c|P(\xi)|
$$

so that

$$
h^{2} \int_{N_{h}^{\prime}}\left(1+\xi^{2}\right)^{2}|\hat{\rho}|^{2} d \xi \leq c^{2} \int_{N_{h}^{\prime}}|P(\xi)|^{2}|\hat{\rho}(\xi)|^{2} d \xi \leq c^{2} \int_{R^{3}}|P(\xi) \hat{\rho}(\xi)|^{2} d \xi=c^{2} \int_{R^{3}}|P(h D) \rho|^{2} d x
$$

If $\xi \in N_{h}$, then one introduces local coordinates in which the set $N$ has the equations

$$
t=0, \quad \xi_{1}=0, \quad t=P_{1}(\xi)
$$

the $\xi_{1}$-axis being chosen along the vector $\mu$ defined by the equation $\beta=m+i \mu$. These local coordinates can be introduced because of the condition (5.9). Define

$$
f:=P_{1}(\xi) \hat{\rho}(\xi)
$$

Then $f=0$ at $t=0$ and $f \in C^{\infty}\left(R^{3}\right)$. Let us use the inequality

$$
\int_{-h}^{h} t^{-2}|f(t)|^{2} d t \leq 4 \int_{-h}^{h}\left|f^{\prime}(t)\right|^{2} d t
$$

proved in Lemma 19 below. This inequality holds for

$$
f \in C^{1}(-h, h), \quad h=\text { const }>0, \quad f(0)=0
$$

Applying (5.24) to (5.23) and integrating (5.24) in the remaining variables $\xi$, one gets

$$
\int_{N_{h}}|\hat{\rho}(\xi)|^{2} d \xi \leq c \int_{N_{h}}\left|\nabla_{\xi}\left[P_{1}(\xi) \hat{\rho}(\xi)\right]\right|^{2} d \xi \leq c \int_{R^{3}}\left|\nabla_{\xi}\left[P_{1}(\xi) \hat{\rho}(\xi)\right]\right|^{2} d \xi
$$

Note that the set $N_{h}$ is bounded. Therefore

$$
h^{2} \int_{N_{h}}\left(1+\xi^{2}\right)^{2}|\hat{\rho}(\xi)|^{2} d \xi \leq c h^{2} \int_{N_{h}}|\hat{\rho}(\xi)|^{2} d \xi
$$


Using Parseval's equality, the assumption supp $\rho \subset B_{r}$, and the S.Bernstein's inequality for derivatives of entire functions of exponential type, one gets

$$
h^{2} \int_{R^{3}}\left|\nabla_{\xi}\left[P_{1}(\xi) \hat{\rho}(\xi)\right]\right|^{2} d \xi=r^{2} \int_{R^{3}}\left|P_{1}(\xi) \hat{\rho}\right|^{2} d x \leq r^{2} \int_{R^{3}}|P(\xi) \hat{\rho}|^{2} d x=r^{2} \int_{R^{3}}|P(h D) \rho|^{2} d x
$$

From (5.26)-(5.28) one obtains

$$
h^{2} \int_{N_{h}}\left(1+\xi^{2}\right)^{2}|\hat{\rho}(\xi)|^{2} d \xi \leq c r^{2} \int_{R^{3}}|P(h D) \rho|^{2} d x
$$

From (5.21) and (5.29) one gets

$$
h^{2} \int_{N_{h}}\left(1+\xi^{2}\right)^{2}|\hat{\rho}(\xi)|^{2} d \xi \leq c^{2} \int_{R^{3}}|P(h D) \rho|^{2} d x
$$

This inequality implies (5.18). Lemma 18 is proved.

Lemma 19: Under the assumptions (5.25) inequality (5.24) holds.

Proof: Inequality (5.24) is similar to the well known Hardy's inequality in which the integration is taken over $(0, \infty)$. To prove $(5.24)$ one starts with an obvious inequality in which $\lambda$ is an arbitrary real number:

$$
0 \leq \int_{-h}^{h}\left|f^{\prime}-\lambda t^{-1} f(t)\right|^{2} d t:=A \lambda^{2}-\lambda B+C
$$

where

$$
A:=\int_{-h}^{h} t^{-2}|f|^{2} d t, \quad B:=\int_{-h}^{h} t^{-1} \frac{d}{d t}|f(t)|^{2} d t
$$

and

$$
C:=\int_{-h}^{h}\left|f^{\prime}\right|^{2} d t
$$

One has

$$
B=\left.t^{-1}|f(t)|^{2}\right|_{-h} ^{h}+\int_{-h}^{h} t^{-2}|f|^{2} d t=\frac{|f(h)|^{2}+|f(-h)|^{2}}{h}+A \geq A
$$

From (5.31) it follows that

$$
B^{2} \leq 4 A C
$$

By (5.34) one has $B \geq A$ and (5.35) implies

$$
A \leq 4 C
$$

This is inequality (5.24). Lemma 19 is proved.

We need one more lemma:

Lemma 20: Let

$$
P(h D) \rho=0 \text { in } A_{1}
$$

where $A_{1}$ is a bounded region with a smooth boundary. Let $A \subset A_{1}, \eta(x) \in C_{0}^{\infty}\left(A_{1}\right), 0 \leq \eta(x) \leq 1, \eta(x)=1$ in $A$, where $A$ is a strictly inner subdomain of $A_{1}$. Then

$$
h\|D \rho\|_{A} \leq c\|\rho\|_{A_{1}}
$$


where $\|\rho\|_{A}:=\|\rho\|_{L^{2}(A)}$.

Proof: Multiply (5.37) by $\eta \bar{\rho}$, take the real part and integrate by parts to get

$$
\begin{aligned}
& h \int_{A_{1}} \eta|\nabla \rho|^{2} d x=-\frac{h}{2} \int_{A_{1}}(\bar{\rho} \nabla \rho+\rho \nabla \bar{\rho}) \nabla \eta d x+2 \operatorname{Re}\left(i \beta_{j} \int_{A_{1}} \rho_{j} \bar{\rho} \eta d x\right)= \\
& =\frac{h}{2} \int_{A_{1}}|\rho|^{2} \nabla^{2} \eta d x+2 \operatorname{Re}\left(i \beta_{j} \int_{A_{1}} \rho_{j} \bar{\rho} \eta d x\right)
\end{aligned}
$$

where the summation over the repeated indices is understood. Using the inequalities

$$
\begin{gathered}
\left|2 \rho_{j} \bar{\rho}\right| \leq \frac{h}{2}\left|\rho_{j}\right|^{2}+2 h^{-1}|\rho|^{2} \\
\left|\nabla^{2} \eta\right| \leq c, \quad\left|\beta_{j}\right| \leq 1
\end{gathered}
$$

one obtains from (5.39) the following inequality

$$
h \int_{A_{1}} \eta|\nabla \rho|^{2} d x \leq c h \int_{A_{1}}|\rho|^{2} d x+\frac{h}{2} \int_{A_{1}} \eta\left|\rho_{j}\right|^{2} d x+2 h^{-1} \int_{A_{1}} \eta|\rho|^{2} d x
$$

It follows from (5.42) that

$$
h^{2} \int_{A}|\nabla \rho|^{2} \leq c_{1} h^{2} \int_{A_{1}}|\rho|^{2} d x+c_{2} \int_{A_{1}} \eta|\rho|^{2} d x \leq c_{3} \int_{A_{1}}|\rho|^{2} d x
$$

This inequality implies (5.38). Lemma 20 is proved.

We are now ready to prove Lemma 6 .

Proof of Lemma 6: The equation for the function $\rho$ defined in (2.12) is

$$
L \rho=q \rho+q \text { in } R^{3}
$$

where $L$ is defined in (5.1) Write (5.44) as

$$
P(h D) \rho=-h^{2}(q \rho+q), \quad h:=|\theta|^{-1}
$$

Let $\eta \in C_{0}^{\infty}\left(B_{b}\right), 0 \leq \eta(x) \leq 1, \eta(x)=1$ in $B_{a_{1}}$. Clearly

$$
P(\eta \rho)=(P \eta-\eta P) \rho-h^{2} \eta(q \rho+q)
$$

Applying (5.18) to (5.46) yields

$$
h\left\|\langle h D\rangle^{2}(\rho \eta)\right\| \leq c\|(P \eta-\eta P) \rho\|+c h^{2}\|q\|_{L^{\infty}\left(B_{a}\right)}\|\rho\|_{a}+c h^{2}\|q\|_{a}
$$

Since $\eta(x)=1$ in $B_{a}$, one obtains

$$
h\|\rho\|_{a} \leq h\left\|\langle h D\rangle^{2}(\eta \rho)\right\| \leq c h^{2}\|\rho\|_{a}+c h^{2}+c\|(P \eta-\eta P) \rho\|
$$

Thus

$$
\|\rho\|_{a} \leq c h+c h^{-1}\|(P \eta-\eta P) \rho\|
$$

Using the equation

$$
D \eta=0 \text { in } B_{a_{1}}
$$


one obtains

$$
\|(P \eta-\eta P) \rho\|=\left\|\rho(h D)^{2} \eta+2 h^{2} D \eta \cdot D \rho+2 h \beta \rho \cdot D \eta\right\| \leq c\left(h^{2}+h\right)\|\rho\|_{a_{1}, b}+c h^{2}\|D \rho\|_{a_{1}, b}
$$

The function $\rho$ solves equation (5.44) and $q(x)=0$ in $B_{b} \backslash B_{a_{1}}$. Therefore Lemma 20 is applicable and the estimate (5.38) yields

$$
h\|D \rho\|_{a_{1}, b} \leq c\|\rho\|_{a_{1}-\varepsilon, b+\varepsilon}
$$

where $\varepsilon>0$ is an arbitrary small number.

From (5.49), (5.51)and (5.52) one obtains

$$
\|\rho\|_{a} \leq c h+c\|\rho\|_{a_{1}-\varepsilon, b+\varepsilon}
$$

Since $\varepsilon>0$ is arbitrarily small, one chooses $\varepsilon$ so small that $a<a_{1}-\varepsilon$, where $a_{1}>a$, and (5.53) implies (2.23). Lemma 6 is proved.

The arguments in this section are close to those in [8].

\section{Proof of Theorems B and C}

We have already proved Theorem A in Section III.1.

Proof of Theorem B: From (2.35) with $\theta=\theta(\delta)$ and $\theta^{\prime}=\theta^{\prime}(\delta)$, where $\theta(\delta), \theta^{\prime}(\delta)$ and $\nu_{\delta}(\alpha)$ are defined in Lemma 8, one has

$$
\hat{q}_{\delta}=-4 \pi \int_{S^{2}}\left[\hat{A}_{\delta}\left(\theta^{\prime}, \alpha\right)-A\left(\theta^{\prime}, \alpha\right)\right] \nu_{\delta}(\alpha) d \alpha-4 \pi \int_{S^{2}} A\left(\theta^{\prime}, \alpha\right) \nu_{\delta}(\alpha) d \alpha:=I_{1}+I_{2}
$$

The last term in (6.1) is transformed as in (3.2) and (3.3) and one obtains the estimate

$$
\left|-4 \pi \int_{S^{2}} A\left(\theta^{\prime}, \alpha\right) \nu_{\delta}(\alpha) d \alpha-\tilde{q}(\lambda)\right| \leq\|q\|_{a}\left\|\rho\left(\nu_{\delta}\right)\right\|_{a} \leq c|\theta|^{-1}
$$

where formula (2.32) was used.

The first term is estimated by (3.5) and (2.32):

$$
\left|I_{1}\right| \leq c a\left(\nu_{\delta}\right) \exp (\kappa b) \mu(\delta) \leq c|\theta|^{-1}
$$

From (6.2) and (6.3) the estimate (2.36) follows.

Let us prove (2.37). To this end one has to estimate the function $\Theta(\delta)$, defined in (2.32), from below. Such an estimate is given in (4.39) and yields (2.37). Finally, Lemma 17 shows that the constants $c$ in our estimates can be chosen uniformly for $q \in \mathcal{B}_{C}$. Theorem B is proved.

Proof of Theorem C: The starting point is formula (2.40). Multiply $(2.40)$ by $\nu_{1}(\alpha, \theta)$ and $\nu_{2}\left(-\alpha^{\prime}, \theta^{\prime}\right)$ and integrate over $S^{2} \times S^{2}$ to get

$$
-4 \pi \int_{S^{2}} \int_{S^{2}} A\left(\alpha^{\prime}, \alpha\right) \nu_{1}(\alpha, \theta) \nu_{2}\left(-\alpha^{\prime}, \theta^{\prime}\right) d \alpha d \alpha^{\prime}=\int_{B_{a}} p(x) \varphi_{1}(x, \theta) \varphi_{2}\left(x, \theta^{\prime}\right) d x
$$

Here $\theta, \theta^{\prime} \in M, \quad|\theta| \gg 1, \theta^{\prime}+\theta=\lambda, \lambda \in R^{3}$,

$$
\varphi_{j}\left(x, \theta_{j}\right):=\int_{S^{2}} u_{j}(x, \alpha) \nu_{j}\left(\alpha, \theta_{j}\right) d \alpha, \quad j=1,2, \quad \theta_{1}:=\theta, \quad \theta_{2}:=\theta^{\prime}
$$

and $\nu_{j}=\nu_{j}\left(\alpha, \theta_{j}\right)$ are chosen so that

$$
\left\|\rho\left(\nu_{j}\right)\right\| \leq c|\theta|^{-1}, \quad \rho\left(\nu_{j}\right)=\exp \left(-i \theta_{j} \cdot x\right) \varphi_{j}-1
$$


where $\left|\theta_{1}\right|\left|\theta_{2}\right|^{-1} \rightarrow 1$, as $\left|\theta_{1}\right| \rightarrow \infty$, and the norm is defined in (2.14). Then, using (6.6) and (2.23), one obtains

$$
\left.\int_{B_{a}} p(x) \varphi_{1} \varphi_{2} d x=\int_{B_{a}} p(x) \exp (i \lambda \cdot x)\left(1+\rho_{1}\right)\left(1+\rho_{2}\right) d x=\tilde{p}(\lambda)+O(|\theta|)^{-1}\right)
$$

Note that for $|\lambda| \leq \lambda_{0}$ one can choose a constant $c$ independent of $\lambda$, such that

$$
O\left(|\theta|^{-1}\right) \leq c|\theta|^{-1}, \quad \frac{\left|\theta_{1}\right|}{\left|\theta_{2}\right|} \rightarrow 1 \text { as }\left|\theta_{1}\right| \rightarrow \infty
$$

The left-hand side of (6.4) can be estimated by the Cauchy inequality. Using (2.41) one gets

$$
\left|-4 \pi \int_{S^{2}} \int_{S^{2}} A\left(\alpha^{\prime}, \alpha\right) \nu_{1} \nu_{2} d \alpha d \alpha^{\prime}\right| \leq 16 \pi^{2} \delta a\left(\nu_{1}\right) a\left(\nu_{2}\right)
$$

From (6.4) and (6.7)-(6.9) one obtains

$$
|\tilde{p}(\lambda)| \leq c\left[\delta a\left(\nu_{1}\right) a\left(\nu_{2}\right)+|\theta|^{-1}\right]
$$

Let us estimate $a\left(\nu_{j}\right)$. If one takes $\varepsilon=\kappa^{-1} \exp (-\kappa b)$ in (3.11), then estimates (6.6) hold (see formulas $(4.7)-(4.9))$. Therefore, by $\left(3.16^{\prime \prime}\right)$,

$$
a\left(\nu_{j}\right) \leq c \kappa^{4}(2 \kappa)^{20 b \kappa}, \quad \kappa=\left|\operatorname{Im} \theta_{1}\right|=\left|\operatorname{Im} \theta_{2}\right|
$$

Thus (6.10) and (6.11) yield

$$
|\tilde{p}(\lambda)| \leq c\left[\delta \kappa^{8}(2 \kappa)^{40 b \kappa}+\kappa^{-1}\right]
$$

For a small fixed $\delta>0$ find the infimum of the right-hand side of (6.12)

$$
\inf _{\kappa>0}\left[\delta \kappa^{8}(2 \kappa)^{40 b \kappa}+\kappa^{-1}\right]:=\eta(\delta)
$$

One has

$$
\delta h(\kappa)+\kappa^{-1}:=\delta \exp (40 b \kappa \ln \kappa+40 b \kappa \ln 2+8 \ln \kappa)+\kappa^{-1}=\inf
$$

Taking the derivative with respect to $\kappa$ yields, at the point of minimum,

$$
\delta h(\kappa)[40 b \ln \kappa+O(1)]=\kappa^{-2}, \quad \kappa \gg 1
$$

Let us solve (6.15) asymptotically, as $\delta \rightarrow 0$, for $\kappa=\kappa(\delta)$. This allows us to estimate $\eta(\delta)$ as $\delta \rightarrow 0$. Taking $\ln$ of $(6.15)$ yields

$$
\ln \frac{1}{\delta}=2 \ln \kappa+40 b \kappa \ln \kappa+40 b \kappa \ln 2+8 \ln \kappa+\ln [40 b \ln \kappa+O(1)]=40 b \kappa(\ln \kappa)[1+o(1)], \quad \kappa \rightarrow 0
$$

This is an equation similar to (4.33). Using the argument given for the proof of Lemma $8^{\prime}$, one obtains the asymptotic solution (6.16). The role of $N(\delta)$ is played by $\ln (1 / \delta)$, and the role of $\gamma_{2}$ is played by $1 /(40 b)$. Thus, (4.35) yields

$$
\kappa(\delta)=\frac{E(\delta)}{\ln E(\delta)}[1+o(1)], \text { where } E(\delta):=\frac{1}{40 b} \ln \frac{1}{\delta}, \quad \delta \rightarrow 0
$$

Equation (6.17) can be written as

$$
\kappa(\delta)=\frac{1}{40 b} \frac{|\ln \delta|}{\ln |\ln \delta|}[1+o(1)], \quad \delta \rightarrow 0
$$


From (6.13)-(6.15) and (6.18) one obtains

$$
\eta(\delta)=\frac{1}{\kappa}[1+o(1)]=40 b \frac{\ln |\ln \delta|}{|\ln \delta|}[1+o(1)] \text { as } \delta \rightarrow 0
$$

This and (6.12) yield (2.42). The constant $c$ by Lemma 17 can be chosen uniformly for $q \in \mathcal{B}_{C}$. Theorem $\mathrm{C}$ is proved.

Theorem C is a refinement and an improvement over the result in [14] (see also [31]).

VII. Summary of the Numerical Procedure, Additional Results and Remarks

In this last section we discuss the following items: a) stability of the recovery of $q(x)$ given $\tilde{q}(\lambda)$ with some error, b) discussion of the steps in a possible numerical implementation of the methods for solving the ISP developed in this paper, and c) an alternative to (2.32) optimization problem which leads algorithmically to a stable approximation of $\tilde{q}(\lambda)$ from the knowledge of noisy data.

1. Suppose $\tilde{q}_{\delta}(\lambda)$ is known such that

$$
\sup _{|\lambda| \leq \lambda_{0}}\left|\tilde{q}_{\delta}(\lambda)-\tilde{q}(\lambda)\right| \leq \delta
$$

where $\lambda_{0}>0$ is a large number, $\tilde{q}(\lambda)$ is defined in (2.20), and a priori it is assumed that $q(x) \in Q$ and

$$
|\tilde{q}(\lambda)| \leq c_{1}\left(1+\lambda^{2}\right)^{-\tilde{d}}, \quad \tilde{d}>\frac{3}{2}, \quad \lambda \in R^{3}
$$

Given $\tilde{q}_{\delta}(\lambda)$ for $|\lambda| \leq \lambda_{0}$, and the numbers $c_{1}, \tilde{d}$ and $\delta$, one wants to estimate $q(x)$ stably as $\delta \rightarrow 0$. Define

$$
q_{\delta}(x):=(2 \pi)^{-3} \int_{|\lambda| \leq \lambda_{0}} \tilde{q}_{\delta}(\lambda) \exp (i \lambda \cdot x) d \lambda
$$

Then

$$
\begin{aligned}
q_{\delta}(x) & =\frac{1}{(2 \pi)^{3}} \int_{|\lambda| \leq \lambda_{0}}\left[\tilde{q}_{\delta}(\lambda)-\tilde{q}(\lambda)\right] \exp (i \lambda \cdot x) d \lambda-\frac{1}{(2 \pi)^{3}} \int_{R^{3} \backslash B_{\lambda_{0}}} \tilde{q}(\lambda) \exp (i \lambda \cdot x) d \lambda+ \\
& +\frac{1}{(2 \pi)^{3}} \int_{R^{3}} \tilde{q}(\lambda) \exp (i \lambda \cdot x) d \lambda:=I_{1}+I_{2}+q(x)
\end{aligned}
$$

One has

$$
\begin{gathered}
\left|I_{1}\right| \leq \delta \lambda_{0}^{3} \frac{1}{6 \pi^{2}} \\
\left|I_{2}\right| \leq \frac{4 \pi c_{1}}{8 \pi^{3}} \int_{\lambda_{0}}^{\infty} \frac{\lambda^{2} d \lambda}{\left(1+\lambda^{2}\right)^{\tilde{d}}}<\frac{c_{1}}{2 \pi^{2}} \int_{\lambda_{0}}^{\infty} \lambda^{-2 \tilde{d}+2} d \lambda=\frac{c_{1}}{2 \pi^{2}} \frac{\lambda_{0}^{-2 \tilde{d}+3}}{2 \tilde{d}-3}
\end{gathered}
$$

From (7.4)-(7.6) one gets

$$
\left|q_{\delta}(x)-q(x)\right| \leq \frac{\delta \lambda_{0}^{3}}{6 \pi^{2}}+\frac{c_{1}}{2 \pi^{2}(2 \tilde{d}-3)} \frac{1}{\lambda_{0}^{2 \tilde{d}-3}}
$$

For a fixed small $\delta$ one can minimize the right-hand side of (7.7) in $\lambda_{0}$ and find $\lambda_{0}(\delta)$ at which the minimum is attained:

$$
\lambda_{0}(\delta)=\left(\frac{c_{1}}{\delta}\right)^{\frac{1}{2 d}}
$$

This equation gives a practical estimate of the region on which $\tilde{q}_{\delta}(\lambda)$ should be known for a stable recovery of $q(x)$. With $\lambda_{0}=\lambda_{0}(\delta)$ given in (7.8) one obtains from (7.7) the following estimate

$$
\left|q_{\delta}(x)-q(x)\right| \leq c_{0} \delta^{1-\frac{3}{2 d}}
$$


where

$$
c_{0}:=\left[\frac{1}{6 \pi^{2}}+\frac{1}{2 \pi^{2}(2 \tilde{d}-3)}\right] c_{1}^{\frac{3}{2 d}}
$$

Let us formulate the result:

Lemma 21: Let (7.1) and (7.2) hold, the numbers $\delta, c_{1}$ and $\tilde{d}$ be known, $\lambda_{0}(\delta)$ be defined in (7.8), and $q_{\delta}(x)$ be given in (7.3). Then the error estimate of the recovery of $q(x)$ by the formula (7.3) is given by (7.9) and (7.10).

2. Let us discuss the steps in a numerical implementation of the methods for solving the $\operatorname{ISP}_{\delta}$ developed in this paper.

Step 1: Given $A_{\delta}\left(\alpha^{\prime}, \alpha\right)$ one calculates $\hat{A}_{\delta}\left(\theta^{\prime}, \alpha\right)$ by formula (2.25), then $u_{\delta}$ and $\rho_{\delta}(\nu)$ by formulas (2.26), (2.27) with $\theta^{\prime}$ and $\theta$ satisfying (2.19).

Step 2: One solves the variational problem (2.32) taking $\theta$ of order given in (2.37). This can be done by minimizing the functional $\left\|\rho_{\delta}(\nu)\right\|$. For example, one can look for $\nu$ of the form

$$
\nu=\sum_{\ell=0}^{n} \nu_{\ell} Y_{\ell}(\alpha)
$$

and find the coefficients $\nu_{\ell}$ from the linear system which one gets from the condition

$$
\left\|\rho_{\delta}(\nu)\right\|=\min
$$

If $\nu_{\delta}(\alpha, \theta)$ is an approximate solution to (7.12), one checks if the inequality (2.32) holds with some constant $c$ independent of $\delta$. This is done by solving several problems with $\delta, \frac{\delta}{2}, \frac{\delta}{4}, \frac{\delta}{8}$ in place of $\delta$. If the inequality (2.32) does not hold, one should increase the number $n$ in (7.11) and try to decrease $|\theta|$.

One may do numerical experiments with some $q(x)$, for example $q(x)=1$ in $B_{a}, q(x)=0$ outside $B_{a}$, and get an idea about the values of $\theta(\delta)$ and $\nu_{\delta}(\alpha)$ needed for recovery. If formulas $(2.38)-\left(2.39^{\prime}\right)$ are used then only discrete noisy data $A_{\delta}\left(\alpha_{p}^{\prime}, \alpha_{j}\right)$ are used for recovery.

3. Let us formulate in conclusion an alternative to (2.32) optimization method for solving the ISP with noisy data. The alternative method consists of the following steps:

1) First, solve the problem

$$
\left\|\rho_{\delta}(\nu)\right\|=\inf , \quad \nu \in L^{2}\left(S^{2}\right)
$$

Denote by $d(\delta, \theta)$ the infimum in (7.13) and find $\nu_{\theta, \delta}(\alpha)$ such that

$$
\left\|\rho_{\delta}\left(\nu_{\theta, \delta}\right)\right\| \leq d(\delta, \theta)+|\theta|^{-1}
$$

2) Secondly, solve the problem

$$
H(\delta, \theta):=\inf :=\omega(\delta), \quad \theta \in M
$$

where

$$
H(\delta, \theta):=\left\|\rho_{\delta}\left(\nu_{\theta, \delta}\right)\right\|+a\left(\nu_{\theta, \delta}\right) \exp (\kappa b) \mu(\delta), \quad \kappa=\operatorname{Im}|\theta|,
$$

and find $\theta_{1}(\delta)$ such that

$$
H\left(\delta, \theta_{1}(\delta)\right) \leq \omega(\delta)+\delta
$$

Define $\hat{q}_{1 \delta}$ by the formula

$$
\hat{q}_{1 \delta}=-4 \pi \int_{S^{2}} \hat{A}\left(\theta_{1}^{\prime}(\delta), \alpha\right) \nu_{1 \delta}(\alpha) d \alpha, \quad \nu_{1 \delta}:=\nu_{\theta_{1}(\delta), \delta}
$$

where $\theta_{1}^{\prime}(\delta)-\theta_{1}(\delta)=\lambda$. Then the following error estimate holds

$$
\sup _{|\lambda| \leq \lambda_{0}}\left|\hat{q}_{1 \delta}-\tilde{q}(\lambda)\right| \leq c\left[\left|\theta_{1}(\delta)\right|^{-1}+\delta+|\theta(\delta)|^{-1}\right]
$$


Here $\theta(\delta)$ satisfies estimate $(2.37)$ and $\theta_{1}(\delta)$ is calculated numerically (see (7.16) and (7.17)).

Let us prove (7.19). First, note that

$$
\left\|\rho\left(\nu_{1 \delta}\right)\right\| \leq c[\omega(\delta)+\delta], \quad c=\text { const } \geq 1
$$

Indeed, by (2.31),

$$
\left\|\rho\left(\nu_{1 \delta}\right)\right\| \leq\left\|\rho_{\delta}\left(\nu_{1 \delta}\right)\right\|+c a\left(\nu_{1 \delta}\right) \exp \left(\kappa_{1} b\right) \mu(\delta) \leq c[\omega(\delta)+\delta], \quad c \geq 1, \quad \kappa_{1}=\left|\operatorname{Im} \theta_{1}\right|
$$

Secondly, as in (6.1)-(6.3), one gets, using Lemma 6, and (3.4),

$$
\left|\hat{q}_{1 \delta}-\tilde{q}(\lambda)\right| \leq c\left[\left\|\rho\left(\nu_{1 \delta}\right)\right\|_{a}+a\left(\nu_{1 \delta}\right) \exp \left(\kappa_{1} b\right) \mu(\delta)\right] \leq c\left[\omega(\delta)+\delta+\left|\theta_{1}(\delta)\right|^{-1}\right]
$$

Thirdly, let us prove that

$$
\omega(\delta) \leq c|\theta(\delta)|^{-1}
$$

where $\theta(\delta)$ is defined in (2.32) and satisfies (2.37). One has, using (7.14),

$$
\omega(\delta) \leq \inf _{\theta \in M}\left[d(\delta, \theta)+|\theta|^{-1}+a\left(\nu_{\theta, \delta}\right) \exp (\kappa b) \mu(\delta)\right] \leq c|\theta(\delta)|^{-1}
$$

where $\theta(\delta)$ satisfies $(2.37)$.

From (7.20), (7.22) and (7.23) estimate (7.19) follows with $|\theta(\delta)|$ satisfying (2.37). Let us summarize the result assuming that $\left|\theta_{1}(\delta)\right| \rightarrow \infty$ as $\delta \rightarrow 0$ :

Lemma 22: The function (7.18) is a stable approximation of $\tilde{q}(\lambda)$ and the error estimate is given in (7.19) with $|\theta(\delta)|$ satisfying inequality (2.37) and $\left|\theta_{1}(\delta)\right|$ defined by (7.17).

The result of Lemma 22 is an improvement over the result in [18].

4. The methods for solving the $\operatorname{ISP}_{\delta}$ developed in this paper are applicable in many fields. For example, the applications to geophysical inverse problems are discussed in [1,6,20,26], to Maxwell's equations in [1], [21], to hyperbolic equations in [1], [22] [30], to inverse conductivity problem in [1],[23-25], to inverse spectral problem in [1],[29] and property $\mathrm{C}$, the basis of our theory, was introduced in [27],[6],[28] and [1].

5. Finally, let us point out that for a study of $\operatorname{ISP}_{\delta}$ it is necessary to assume that $q(x)$ vanishes outside some ball. Indeed, if $q(x) \in L^{1}\left(R^{3}\right)$ then the function

$$
q_{a}(x)= \begin{cases}q(x), & \text { if }|x|>a \\ 0, & \text { if }|x|<a\end{cases}
$$

contributes to the scattering amplitude

$$
A\left(\alpha^{\prime}, \alpha\right)=-(4 \pi)^{-1} \int_{R^{3}} \exp \left(-i \alpha^{\prime} \cdot x\right) u(x, \alpha) q(x) d x
$$

the quantity which does not exceed

$$
(4 \pi)^{-1} c \int_{|x| \geq a}|q(x)| d x \rightarrow 0 \text { as } a \rightarrow \infty,
$$

where $c$ is the constant in (3.61). This contribution becomes indistinguishable from the noise when

$$
\int_{|x| \geq a}|q(x)| d x<4 \pi c^{-1} \delta
$$

Thus, one cannot recover $q(x)$ in the region $|x|>a$, where $a$ is determined by (7.25). 
From (7.25) one can estimate the order of the radius $a$ of the ball in which one can recover the potential given the noisy data with the noise level $\delta$. For example, if one knows a priori that $|q(x)| \leq c_{1}|x|^{-d^{\prime}}, d^{\prime}>3$, $|x|>1$, then $(7.25)$ implies $a^{3-d^{\prime}}<\left(\left(d^{\prime}-3\right) / c_{1} c\right) \delta$, so that $a>\left(d^{\prime}-3\right)\left(c_{1} c\right)^{-1} \delta^{1 /\left(d^{\prime}-3\right)}$.

Acknowledgements: The author thanks ONR, NSF and USIEF for support, Prof. J. Sjöstrand whose ideas were crucial in the proof of Lemma 6, and Dr. P. Stefanov for correspondence. This paper was written while the author was a Fulbright Research Professor at the Technion.

\section{REFERENCES}

[0] L. Hörmander, The Analysis of of Linear Partial Differential Operators, Vol. 1-4, Springer-Verlag, New York, 1983-1985.

[1] A. G. Ramm, Multidemensional Inverse Scattering Problems, Longman, New York, 1992.(Expanded Russian edition Mir Publishers, Moscow, 1994, pp.1-496).

[2] A. G. Ramm, Random Fields Estimation Theory, Longman, New York, 1990.(Expanded Russian edition Mir Publishers, Moscow, 1996).

[3] A. G. Ramm, Scattering by Obstacles, D. Reidel, Dordrecht, 1986.

[4] A. G. Ramm, Recovery of the potential from fixed-energy scattering data, Inverse problems 4 (1988), $877-886 ; 5$ (1989), 255.

[5] A. G. Ramm, Stability of the numerical method for solving 3D inverse scattering problem with fixedenergy data, Inverse Problems 6 (1990), L7-L12; J. fuer die reine und angewandte Math.,414, (1991),121.

[6] A. G. Ramm, Multidimensional inverse problems and completeness of the products of solution to PDE, J. Math. Anal. Appl. 134 (1988), 251-253; 136 (1988), 568-574; 139 (1989), 302.

[7] A. G. Ramm, Stability of the inversion of 3D fixed-frequency data, J. Math. Anal. Appl. œebf 169 N2,(1992), 329-349.

[8] A. G. Ramm, Stability of the solution to 3D inverse scattering problem, J. Math. Anal. Appl. œebf 170 N1, (1992),1-15.

[9] A. G. Ramm, Completeness of the products of solutions to PDE and inverse problems, Inverse Problems 6 (1990), 635-664.

[10] A. G. Ramm, Property C and inverse problems, ICM-90 Satellite Conference Proceedings, Inverse Problems in Engineering Sciences, Proc. of a conference held in Osaka 1990, pp. 139-144.

[11] A. G. Ramm, Exact inversion of fixed-energy data, in the book Mathematical and Numerical Aspects of Wave Propagation Phenomena, SIAM, Philadelphia 1991, pp. 481-486.

[12] A. G. Ramm, Numerical method for solving 3D inverse problems with complete and incomplete data, in the book Wave Phenomena, Springer-Verlag, New York 1989, pp. 34-43 (ed. L. Lam and H. Morris).

[13] A. G. Ramm, An approximation problem, Appl. Math. Lett. 4, N5 (1991), 75-77.

[14] A. G. Ramm, Stability of the solution to the inverse scattering problem with exact data, Appl. Math. Lett. 5, N1 (1992), 91-94.

[15] A. G. Ramm, Numerical recovery of the 3D potential from fixed-energy incomplete scattering data, Appl. Math. Lett. 2, N1 (1989), 101-104.

[16] A. G. Ramm, Numerical solution of 3D inverse scattering problems with noisy discrete fixed-energy data, Appl.Math.Lett., 5,N6 (1992),15-18.

[17] A. G. Ramm, Approximation by the scattering solutions and applications to inverse scattering Math. and Comp. Modelling, 18, N1 (1993), 47-56.

[18] A. G. Ramm, Inverse scattering with fixed-energy data Appl.Math.Lett.,5,N4 (1992),63-67.

[19] A. G. Ramm, Spectral properties of the Schrödinger operator in some infinite domains, Matem. Sborn. 66 (1965), 321-343.

[20] A. G. Ramm, Numerical method for solving 3D inverse problems of geophysics, J. Math. Anal. Appl., 136 (1988), 352-356.

[21] A. G. Ramm, An inverse problem for Maxwell's equations, Phys. Lett. 138A (1989), 459-462.

[22] A. G. Ramm and Rakesh, Property C and an inverse problem for a hyperbolic equation, J. Math. Appl. 156 (1991), 209-219.

[23] A. G. Ramm, A simple proof of uniqueness theorem in impedance tomography, Appl. Math. Lett. 1 N3 (1988), 381-384. 
[24] A. G. Ramm, Multidimensional inverse problems and completeness of the products of homogenous PDE, Zeitschr. f. Angew. Math. u. Mech. 69, N3 (1989), T13-T21.

[25] A. G. Ramm, Finding conductivity from boundary measurements, Comp. and Math. with Appl. 21, N8 (1991), 85-91.

[26] A. G. Ramm, Uniqueness theorems for geophysical problems with incomplete surface data, Appl. Math. Lett. 3, N4 (1990), 41-44.

[27] A.G.Ramm, On completeness of the products of harmonic functions, Proc.Amer.Math.Soc., 99, (1986), 253-256.

[28] A.G.Ramm, Necessary and sufficient condition for a PDO to have property C, J. Math. Anal. Appl. 156, (1991),505-509.

[29] A.G.Ramm, Property C and uniqueness theorems for multidimensional inverse spectral problem, Appl. Math. Lett. 3, (1990),57-60.

[30] A.G.Ramm, J.Sjöstrand, An inverse problem for the wave equation,Math.Zeit.,206, (1991), 119-130.

[31] P. Stefanov, Stability of the inverse problem in potential scattering at fixed energy, Ann. Inst. Fourier, Grenoble 40, N4 (1990), 867-884.

email: ramm@math.ksu.edu 